\title{
Asymptotics for a censored generalized linear model with unknown link function
}

\author{
Yanhua Wang • Shuyuan He • Lixing Zhu • \\ Kam C. Yuen
}

Received: 16 October 2004 / Revised: 24 May 2006 /

Published online: 23 August 2006

(C) Springer-Verlag 2006

\begin{abstract}
For censored response variable against projected co-variable, a generalized linear model with an unknown link function can cover almost all existing models under censorship. Its special cases include the accelerated failure time model with censored data. Such a model in the uncensored case is called the single-index model in econometrics. In this paper, we systematically study the asymptotic properties. We derive the central limit theorem and the law of the iterated logarithm for an estimator of the direction parameter. We also obtain the optimal convergence rate of an estimator of the unknown link function in the model.
\end{abstract}

Keywords Central limit theorem - Projection pursuit regression - Random censorship · Generalized linear model · Unknown link

Mathematics Subject Classification (2000) Primary 62H99; Secondary 62H05

Y. Wang $\cdot$ S. He

Peking University, Beijing, China

L. Zhu ( $\otimes)$

Department of Mathematics, Hong Kong Baptist University, Kowloon Tong, Road,

Hong Kong, China

e-mail: lzhu@hkbu.edu.hk

L. Zhu

East China Normal University, Shanghai, China

K. C. Yuen

The University of Hong Kong, Hong Kong, China 


\section{Introduction}

Dimension-reduction techniques have received much attention in the literature. As a dimension-reduction method, single-index models have been studied extensively in recent years. They are regarded as a special case of projection pursuit regression (PPR) termed by Friedman and Stuetzle [7] whose estimation procedure has been further studied by Huber [17]. Hall [10] derived optimal convergence rates of projection pursuit estimators and Zhu and Fang [31] obtained the same rates under milder conditions. For single-index models, Ichimura [19] achieved the asymptotic normality of the parameter estimator and Härdle and Stoker [11] obtained the convergence rate of $n^{r / 2 r+1}$ of the estimator of link function $g$ which is $r$-order differentiable. Härdle et al. [12] also verified the asymptotic normality when an optimal bandwidth is adopted. Horowitz and Härdle [16] studied the semiparametric estimation of single-index models with discrete covariates. Daniela et al. [4] proposed two automatic rules for estimating the direction parameter and the smoothing parameter in the single index Poisson model. Delecroix et al. [5] examined an asymptotically efficient estimator via pseudo-maximum likelihood method. In addition, Li and Duan [23], Ichimura [20], Carroll et al. [2], Xia et al. [29] and Huh and Park [18] also performed various investigations of generalized single index models.

In the literature, not much work has been done for single-index models with censored response variable. To the best of our knowledge, for a random censorship model with possibly multi-indices, Li et al. [24] studied the estimation of indices using sliced inverse regression (SIR). Their estimator is root- $n$ consistent under certain conditions. It is however that their estimation procedure requires a kernel smoothing for high-dimensional data which is inconsistent with the goal of dimension reduction. Another related work was given by Stute [26] who obtained an asymptotically normal estimator of the parameter in a non-linear censored model.

Suppose that $X$ and $Y$ satisfy the following relation

$$
Y=g\left(\theta_{0}^{\tau} X\right)+\varepsilon,
$$

where $Y$ is the response variable with distribution function $F, X=\left(X_{1}, X_{2}, \ldots\right.$, $\left.X_{p}\right)^{\tau}$ is a $p$-dimensional random vector of covariables, the link function $g(x)$ is an unknown univariate real function, and $\varepsilon$ is a random error with $E(\varepsilon \mid X)=0$. Note that the superscript " $\tau$ " stands for transpose. It is assumed that the direction parameter $\theta_{0} \in \mathbb{R}^{p}$ is unity in $L_{2}$-norm, that is, $\left\|\theta_{0}\right\|_{2}=1$. Denote the joint distribution function of $(X, Y)$ by $F^{0}(x, y)$. Furthermore, for the identifiability of $\theta_{0}$, we need to restrict, without loss of generality, the first component of $\theta_{0}$ to be nonnegative.

Under the random censorship model, the response variable $Y$ is at risk of being censored from the right by a random variable $C$ having distribution function $G$. Hence, we observe $(U, X, \delta)$ where $U=\min (Y, C)$ and $\delta=I(Y \leq C)$ with $I(A)$ being the indicator function of $A$. Assume that $(X, Y)$ and $C$ are independent. 
Let $\bar{G}(x-)=1-G(x-)$ where $G(x-)$ denotes the left continuous version of $G(x)$. Let $b_{F}=\sup \{x: F(x)<1\}$ and $b_{G}=\sup \{x: G(x)<1\}$. Since $b_{F} \leq b_{G}$ is necessary and sufficient for $F^{0}\left(x, y \wedge b_{G}\right)=F^{0}(x, y)$ with $u \wedge v=\min (u, v)$, we assume $b_{F} \leq b_{G}$. For any real function $\phi(x) \neq 0$, let $\phi^{-1}(x)=1 / \phi(x)$.

Following the idea of Koul et al. [21] (hereinafter referred to as KSV), we obtain

$$
\mathbf{E}\left(\frac{\delta U}{\bar{G}(U-)} \mid X\right)=g\left(\theta_{0}^{\tau} X\right) .
$$

Let $T=T(U, \delta, G)=\delta U \bar{G}^{-1}(U-)$. Then, $(U, X, \delta)$ satisfy the following singleindex model

$$
T(U, \delta, G)=g\left(\theta_{0}^{\tau} X\right)+\varepsilon^{*},
$$

where $\mathbf{E}\left(\varepsilon^{*} \mid X\right)=0$. If $G$ is known, model (1.2) is essentially a single-index model with complete data so that the results of Härdle et al. [12] can be applied directly to obtain the asymptotic normality. When $G$ is unknown, the model involves three unknowns $\theta_{0}, g$ and $G$. The estimation becomes more complex in this case. In this paper, we focus on deriving asymptotic results for the estimation with unknown $G$. Applying an optimal bandwidth, we obtain the central limit theorem (CLT), for an estimator of $\theta_{0}$, and the optimal convergence rate of an estimator of $g$. It is worthwhile to point out that such an extension from non-censoring to censoring is not trivial.

More generally, following the idea of Fan and Gijbels [6], one can extend the KSV transformation to a class of transformation:

$$
\phi_{1}(y)=(1+\beta) \int_{0}^{y} \frac{\mathrm{d} t}{\bar{G}(t)}-\beta \frac{y}{\bar{G}(y)}, \quad \text { and } \quad \phi_{2}(y)=(1+\beta) \int_{0}^{y} \frac{\mathrm{d} t}{\bar{G}(t)},
$$

where for simplicity we work with nonnegative random variables. Note that the KSV transformation corresponds to $\beta=-1$ and that the Leurgans [22] transformation relates to $\beta=0$. The tuning parameter $\beta$ in this new class of transformations (1.3) creates the opportunity to improvement. The choice $\beta>0$ focuses more on the censored observations than on the uncensored observations, which is more intuitive than Leurgans's equal choice.

Replacing the data point $(X, U, \delta)$ with the transformed data point $\left(X, T^{*}\right)$ according to $T^{*} \equiv T^{*}(U, \delta, G)=\delta \phi_{1}(U)+(1-\delta) \phi_{2}(U)$, we get $\mathbf{E}\left(T^{*} \mid X\right)=$ $g\left(\theta_{0}^{\tau} X\right)$. Then, $\left(X, T^{*}\right)$ satisfy the following single-index model

$$
T^{*}=g\left(\theta_{0}^{\tau} X\right)+\varepsilon^{* *},
$$

where $\mathbf{E}\left(\varepsilon^{* *} \mid X\right)=0$. In this paper, we also derive asymptotic results for the estimation with unknown $G$. Applying an optimal bandwidth, we obtain 
CLT for an estimator of $\theta_{0}$ as well as the optimal convergence rate of an estimatorof $g$.

Section 2 presents the estimation procedure of model (1.2) and the main results are stated in Sect. 3. Section 4 gives the extended estimation procedure of model (1.4) and the corresponding results. As the proofs are quite long, we arrange them into four Appendices. Appendix A gives the proofs of the main results which require other results derived in Appendices B, C and D.

\section{Estimation procedure}

Write $X_{i}=\left(X_{i 1}, X_{i 2}, \ldots, X_{i p}\right)^{\tau}$. Our observations are $\left\{\left(U_{i}, X_{i}, \delta_{i}\right) ; i=1,2, \ldots, n\right\}$, which is a random sample from the population $(U, X, \delta)$. Throughout the paper, we assume $\left(U_{i}, X_{i}, \delta_{i}\right), i=1, \ldots, n$ are i.i.d. and $C_{1}, \ldots, C_{n}$ are i.i.d. random variables which are independent of i.i.d. random variables $\left(X_{1}, Y_{1}\right), \ldots,\left(X_{n}, Y_{n}\right)$.

Let $U_{1, n} \leq U_{2, n} \leq \cdots \leq U_{n, n}$ be the ordered values of $U$ 's among the first $n$ observations, where ties among lifetimes and censoring times are treated as if the former precedes the later. Let $\delta_{i, n}$ and $X_{i, n}$ be the concomitant of the $U_{i, n}$ for $i=1, \ldots, n$.

In model (1.2), we replace $G$ with the time-honored Kaplan-Meier estimator $G_{n}$ defined by

$$
1-G_{n}(x)=\prod_{U_{i, n} \leq x}\left(1-\frac{1}{n-i+1}\right)^{1-\delta_{i, n}}
$$

which depends on neither $\theta_{0}$ nor $g$. And then, for $j=1, \ldots, n$,

$$
\frac{1}{\bar{G}_{n}\left(U_{j, n}-\right)}=\frac{n}{n-j+1} \prod_{i=1}^{j-1}\left(\frac{n-i}{n-i+1}\right)^{\delta_{i, n}}
$$

where $\bar{G}_{n}(x-)=1-G_{n}(x-)$ is the left continuous version of $G_{n}(x)$.

We then use the Nadaraya-Watson kernel approach to estimate $\theta_{0}$ and $g$. Consider $g(u)=\mathbf{E}\left(Y \mid \theta_{0}^{\tau} X=u\right)=\mathbf{E}\left(T \mid \theta_{0}^{\tau} X=u\right)$. Define $g(u \mid \theta)=\mathbf{E}\left(T \mid \theta^{\tau}\right.$ $X=u)$ and

$$
\hat{g}_{\hat{T}_{i}}(u \mid \theta)=\frac{\sum_{j \neq i} \hat{T}_{j} K_{h}\left(u-\theta^{\tau} X_{j}\right)}{\sum_{j \neq i} K_{h}\left(u-\theta^{\tau} X_{j}\right)} \quad \text { with } \hat{T}_{j}=\frac{\delta_{j} U_{j}}{1-G_{n}\left(U_{j}-\right)},
$$

for any fixed $\theta$, where the kernel function $K$ is a symmetric probability density function with support on $(-1,1)$, and $h$ is a bandwidth. Write $K_{h}(\cdot)=K\left(\cdot h^{-1}\right)$. It is clear that $\hat{g}_{\hat{T}_{i}}(u \mid \theta)$ is the 'delete-one' kernel estimator of $g(u \mid \theta)$ for any fixed $\theta$. 
In order to define an estimator of $\theta_{0}$ and select a bandwidth $\hat{h}_{n}$, we consider

$$
S_{n}(\theta, h)=\sum_{i=1}^{n}\left(\hat{T}_{i}-\hat{g}_{\hat{T}_{i}}\left(\theta^{\tau} X_{i} \mid \theta\right)\right)^{2} I\left(X_{i} \in \mathcal{A}\right) .
$$

Here, we choose $\mathcal{A} \subset \mathbb{R}^{p}$ so that the denominator of the kernel estimator $\hat{g}_{\hat{T}_{i}}(u \mid \theta)$ is not too close to 0 for all $\theta \in \Theta$ to avoid the boundary problem.

Under the help of the existing results obtained by Li et al. [24], we can define the estimators of $\theta_{0}$ and choose a bandwidth $h_{n}$. The estimators are of good asymptotic properties. When the model studied by Li et al. [24] is reduced to model (1.1), the estimator $\theta_{n}^{\prime}$ is of root $n$ consistency to $\theta_{0}$. In other words, $\theta_{n}^{\prime}$ lies within the domain $\Theta_{n}=\left\{\theta \in \Theta \mid\left\|\theta-\theta_{0}\right\|_{2} \leq C_{0} n^{-\frac{1}{2}+\alpha}, \theta_{0} \in \Theta\right\}$ for some $C_{0}>0,0<\alpha<20^{-1}$. Also $\theta_{0} \in \Theta_{n}^{\prime}=\left\{\theta \in \Theta \mid\left\|\theta-\theta_{n}^{\prime}\right\|_{2} \leq C_{0} n^{-\frac{1}{2}+\alpha}, \theta_{0} \in \Theta\right\}$. Therefore, if we use $\theta_{n}^{\prime}$ as an initial estimator, we can define the resultant estimators $\left(\theta_{n}, h_{n}\right)$ as the minimizers of $S_{n}(\theta, h)$ over a much smaller domain of $\theta$ and $h$. For the sake of notational simplicity, we write $\Theta_{n}^{\prime}$ as $\Theta_{n}$. Let $\mathcal{H}_{n}=$ $\left\{h \mid C_{1} n^{-\frac{1}{5}} \leq h \leq C_{2} n^{-\frac{1}{5}}\right\}$ for some $0<C_{1}<C_{2}<\infty$. Let $\left(\hat{\theta}_{n}, \hat{h}_{n}\right)$ minimize $S_{n}(\theta, h)$ over $\Theta_{n} \times \mathcal{H}_{n}$. Denote the estimator of $\theta_{0}$ by $\hat{\theta}_{n}$ and choose $\hat{h}_{n}$ as the bandwidth for use.

Then, define

$$
\hat{g}_{\hat{T}_{i}}\left(u \mid \hat{\theta}_{n}\right)=\frac{\sum_{j \neq i} \hat{T}_{j} K_{\hat{h}_{n}}\left(u-\hat{\theta}_{n}^{\tau} X_{j}\right)}{\sum_{j \neq i} K_{\hat{h}_{n}}\left(u-\hat{\theta}_{n}^{\tau} X_{j}\right)}
$$

as an estimator of $g(u)$.

Remark 2.1 In the estimation procedure, if we use an optimal bandwidth, the defined estimators of $g$ and $\theta_{0}$ will have nice asymptotic properties. For this, we define a theoretic optimal bandwidth $h_{0}$ which minimizes

$$
J(h)=\mathbf{E}\left(\hat{g}_{T_{i}}\left(\theta_{0}^{\tau} X_{i} \mid \theta_{0}\right)-g\left(\theta_{0}^{\tau} X_{i}\right)\right)^{2} I\left(X_{i} \in \mathcal{A}\right),
$$

over all $h$, where

$$
\hat{g}_{T_{i}}(u \mid \theta)=\frac{\sum_{j \neq i} T_{j} K_{h}\left(u-\theta^{\tau} X_{j}\right)}{\sum_{j \neq i} K_{h}\left(u-\theta^{\tau} X_{j}\right)}, \quad \text { here } \quad T_{j}=\frac{\delta_{j} U_{j}}{1-G\left(U_{j}-\right)}
$$

Under some regularity conditions, we will prove that the chosen $\hat{h}_{n}$ is the asymptotically equivalent optimal bandwidth, that is, $\hat{h}_{n} h_{0}^{-1} \rightarrow 1$ in probability as $n \rightarrow \infty$. 


\section{Asymptotic behavior of the estimators}

Assume that $X$ has a support $\mathcal{C} \subset \mathbb{R}^{p}$. Denote the density function of $X$ and $\theta_{0}^{\tau} X$ by $f$ and $v$, respectively. Given $a>0$, let $\mathcal{A}=\left\{x \in \mathcal{C} \mid v\left(\theta_{0}^{\tau} x\right) \geq a\right\}$. Given $\delta>0$, let $\mathcal{A}^{\delta}=\left\{x \in \mathcal{C} \mid \exists y \in \mathcal{A},\|x-y\|_{2} \leq \delta\right\}$ and $\mathcal{B}=\left\{\theta_{0}^{\tau} x \mid x \in \mathcal{A}^{\delta}\right\}$. Define indices sets $\Lambda_{A}=\left\{i \mid X_{i} \in \mathcal{A}\right\}$. Let $\Theta$ be the set of all unit $p$-vectors whose first component is nonnegative. For notational convenience, write $\Omega_{n}=\mathcal{A}^{\delta} \times \Theta_{n} \times \mathcal{H}_{n}$ and $\bar{\Omega}_{n}=\mathcal{C} \times \Theta_{n} \times \mathcal{H}_{n}$.

Usually, $\mathcal{A}$ is dependent on the initial estimator of $\theta_{0}$ or $\theta_{0}$ itself. In contrast, when $\theta^{\tau} X$ is identically distributed for all $\theta \in \Theta, \mathcal{A}$ is free of $\theta_{0}$. This is the case when the distribution of $X$ is elliptically symmetric. Thus, we can easily choose $\mathcal{A}$ by the quantile of the empirical distribution of $\theta^{\tau} X$ with known $\theta \in \Theta$ such as $\theta=p^{-\frac{1}{2}}(1, \ldots, 1)$ in our implementations. Also $\mathcal{A}$ is related to the truncation constant $a$ that is used to bound the density away from zero. In practice, we may choose it such that we have, say, $95 \%$ of data to be used for estimation. In other words, we only trim $5 \%$ of data off from the tails. Define

$$
\vec{\mu}(t \mid \theta)=\mathbf{E}\left(X \mid \theta^{\tau} X=t\right) \quad \text { and } \quad \vec{\mu}(t)=\mathbf{E}\left(X \mid \theta_{0}^{\tau} X=t\right) .
$$

For the derivations of the main results, we need the following regularity conditions labeled by $\mathcal{L}$ :

(L1) $f$ is bounded away from 0 on $\mathcal{A}^{\delta}$ for some $\delta>0$;

(L2) $g$ and $v$ have second-order bounded and continuous derivatives on $\mathcal{B}$;

(L3) $\vec{\mu}(t \mid \theta)$ has second-order bounded and continuous partial derivatives with respect to $\theta$ for all $\theta \in \Theta$ and $t \in \mathcal{B}$, and $\vec{\mu}(t)$ has second-order bounded and continuous derivatives on $\mathcal{B}$;

(L4) $K$ has a support on the interval $(-1,1)$ and is a symmetric probability density with second-order bounded derivatives;

(L5) $F$ and $G$ are continuous, and $b_{F} \leq b_{G}$;

(L6) $\int_{-\infty}^{b_{F}} \bar{F}^{-1} \mathrm{~d} G<\infty$ where $\bar{F}=1-F$;

(L7) $\mathbf{E}\left(\left(1+\varepsilon^{2}\right) \bar{G}^{-1}(Y) \mid X=x\right)$ is bounded.

(L8) $\mathbf{E}\left(\left(1+|\varepsilon|^{m}\right) \bar{G}^{1-m}(Y)\right)<\infty$ for some enough large $m>0$.

Remark 3.1 The above regularity conditions are mild. Condition (L1) is to avoid the boundary problem when a nonparametric regression estimator is applied. This is a commonly-used condition. Conditions (L2) and (L3) are for the nonparametric convergence rate of the estimator. The use of second-order kernel specified in (L4) is associated with (L2) and (L3). Conditions (L5)-(L8) are designed for right censoring. It is clear that Condition (L5) is a commonlyused condition but it could be relaxed slightly in some simpler situations (see $[15,25,27])$. Condition (L6) ensures the weak convergence for the KaplanMeier estimator $G_{n}$ of $G$. Condition (L7) is equivalent with $\mathbf{E}\left(\left|\varepsilon^{*}\right|^{2} \mid X=x\right)$ is bounded. Condition (L8) is equivalent with $\mathbf{E}\left(\left|\varepsilon^{*}\right|^{m}\right)<\infty$ for some $m>0$. If $b_{F}<b_{G}$, (L7) reduces to $\mathbf{E}\left(\varepsilon^{2} \mid X=x\right)$ is bounded, and (L8) reduces to $\mathbf{E}\left(|\varepsilon|^{m}\right)<\infty$ for some $m>0$. Note that if $G$ is known in model (1.2), 
conditions in $\mathcal{L}$ except (L5)-(L6) are the same as those in $\mathcal{L}$ of Härdle et al. [12]. Also, if there is no censoring $(G(x) \equiv 0)$ for model (1.1), conditions in $\mathcal{L}$ except (L5)-(L6) reduce to those in $\mathcal{L}$ of Härdle et al. [12].

Define

$$
\begin{aligned}
W & =\mathbf{E}\left(\left(X-\vec{\mu}\left(\theta_{0}^{\tau} X\right)\right)\left(X-\vec{\mu}\left(\theta_{0}^{\tau} X\right)\right)^{\tau} g^{\prime}\left(\theta_{0}^{\tau} X\right)^{2} I(X \in \mathcal{A})\right), \\
V & =\int \sigma^{* 2}(x) \vec{h}_{\mathcal{A}}(x) \vec{h}_{\mathcal{A}}^{\tau}(x) f(x) \mathrm{d} x-\int \frac{\vec{\psi}_{\mathcal{A}}(s) \vec{\psi}_{\mathcal{A}}^{\tau}(s)}{\bar{F}(s) \bar{G}^{2}(s)} \mathrm{d} G(s), \\
\sigma^{* 2}(x) & =\mathbf{E}\left(\left(\varepsilon^{*}\right)^{2} \mid X=x\right)=\mathbf{E}\left(\frac{G(Y)}{\bar{G}(Y)} Y^{2} \mid X=x\right)+\sigma^{2}(x), \\
\vec{h}_{\mathcal{A}}(x) & =\left(x-\vec{\mu}\left(\theta_{0}^{\tau} x\right)\right) g^{\prime}\left(\theta_{0}^{\tau} x\right) I(x \in \mathcal{A}), \\
\vec{\psi}_{\mathcal{A}}(s) & =\int_{y \geq s} y \vec{h}_{\mathcal{A}}(x) F^{0}(\mathrm{~d} x, \mathrm{~d} y),
\end{aligned}
$$

where $\vec{\mu}\left(\theta_{0}^{\tau} x\right)$ and $\varepsilon^{*}$ are given in (3.1) and (1.2), respectively.

We now present the main results of the paper. Their proofs are given in Appendix A.

Theorem 3.1 Under $\mathcal{L}$, we have

$n^{\frac{1}{2}}\left(\hat{\theta}_{n}-\theta_{0}\right) \rightarrow N\left(0, W^{-} V W^{-}\right) \quad$ in distribution, $\quad$ and $\quad \frac{\hat{h}_{n}}{h_{0}} \rightarrow 1 \quad$ in probability,

where $V$ is given in (3.3) and $W^{-}$denotes the generalized inverse of $W$ in (3.2).

Remark 3.2 Without censoring, the asymptotic co-variance matrix $W^{-} V W^{-}=$ $W^{-} V_{0} W^{-}$, where $V_{0}=\int \sigma^{2}(x) \vec{h}_{\mathcal{A}}(x) \vec{h}_{\mathcal{A}}^{\tau}(x) f(x) \mathrm{d} x$ with $\sigma^{2}(x)=\mathbf{E}\left(\varepsilon^{2} \mid X=x\right)$ given in Härdle [12]. By Hölder's inequality, one can show that $W^{-} V W^{-}-$ $W^{-} V_{0} W^{-}$is positive semi-definite. The asymptotic co-variance matrix $W^{-} V W^{-}$ can be consistently estimated by its corresponding empirical version since

$$
V=\mathbf{E}\left(\left(\frac{\delta U}{\bar{G}(U)}-g\left(\theta_{0}^{\tau} X\right)\right)^{2} \vec{h}_{\mathcal{A}}(X) \vec{h}_{\mathcal{A}}^{\tau}(X)\right)-\mathbf{E}\left(\frac{(1-\delta) \vec{\psi}_{\mathcal{A}}(U) \vec{\psi}_{\mathcal{A}}^{\tau}(U)}{\bar{H}^{2}(U)}\right)
$$

where $\bar{H}(x)=1-H(x)=P(U>x)$ and $\vec{\psi}_{\mathcal{A}}(s)=\mathbf{E}\left(\delta U I(U \geq s) \bar{G}^{-1}(U)\right)$.

Theorem 3.2 Under $\mathcal{L}$ except $(L 8)$, if $\boldsymbol{E}\left(\left(1+|\varepsilon|^{3}\right) \bar{G}^{-3}(Y)\right)<\infty$, we have $\sup _{u \in\left\{\theta^{\tau} x \mid(x, \theta) \in \mathcal{A}^{\delta} \times \Theta_{n}\right\}}\left|\hat{g}_{\hat{T}_{i}}\left(u \mid \hat{\theta}_{n}\right)-g(u)\right|=O_{p}\left(n^{-\frac{2}{5}}(\ln n)^{\frac{1}{2}}\right)$, where $\hat{g}_{\hat{T}_{i}}\left(u \mid \hat{\theta}_{n}\right)$ and $g(u)$ are defined in (2.3) and (1.1), respectively. 


\section{Estimation procedure for a family of transformation and asymptotic behavior of the estimators}

In model (1.4), we replace $G$ with the Kaplan-Meier estimator $G_{n}$ which depends on neither $\theta_{0}$ nor $g$. We now use the Nadaraya-Watson kernel approach to estimate $\theta_{0}$ and $g$. Define

$$
\hat{g}_{\hat{T}_{i}^{*}}(u \mid \theta)=\frac{\sum_{j \neq i} \hat{T}_{j}^{*} K_{h}\left(u-\theta^{\tau} X_{j}\right)}{\sum_{j \neq i} K_{h}\left(u-\theta^{\tau} X_{j}\right)},
$$

where

$$
\hat{T}_{j}^{*}=\delta_{j}\left((1+\beta) \int_{0}^{Y_{j}} \frac{\mathrm{d} t}{\bar{G}_{n}(t-)}-\beta \frac{Y_{j}}{\bar{G}_{n}\left(Y_{j}-\right)}\right)+\left(1-\delta_{j}\right)(1+\beta) \int_{0}^{C_{j}} \frac{\mathrm{d} t}{\bar{G}_{n}(t-)} .
$$

To define an estimator of $\theta_{0}$ and to select a bandwidth $\hat{h}_{n}$ for use, we consider

$$
S_{n}(\theta, h)=\sum_{i=1}^{n}\left(\hat{T}_{i}^{*}-\hat{g}_{\hat{T}_{i}^{*}}\left(\theta^{\tau} X_{i} \mid \theta\right)\right)^{2} I\left(X_{i} \in \mathcal{A}\right) .
$$

Let $\left(\tilde{\theta}_{n}, \tilde{h}_{n}\right)$ minimize $S_{n}(\theta, h)$ over $\Theta_{n} \times \mathcal{H}_{n}$. Denote the estimator of $\theta_{0}$ by $\tilde{\theta}_{n}$ and choose $\tilde{h}_{n}$ as the bandwidth for use. Then, define

$$
\hat{g}_{\hat{T}_{i}^{*}}\left(u \mid \tilde{\theta}_{n}\right)=\frac{\sum_{j \neq i} \hat{T}_{i}^{*} K_{\tilde{h}_{n}}\left(u-\tilde{\theta}_{n}^{\tau} X_{j}\right)}{\sum_{j \neq i} K_{\tilde{h}_{n}}\left(u-\tilde{\theta}_{n}^{\tau} X_{j}\right)}
$$

as an estimator of $g(u)$.

Remark 4.1 For implementation, we may take

$$
\hat{\beta}=\min _{\left\{i: \delta_{i}=1\right\}} \frac{\int_{0}^{Y_{i}} \frac{\mathrm{d} t}{\bar{G}(t)}-Y_{i}}{\frac{Y_{i}}{\bar{G}\left(Y_{i}\right)}-\int_{0}^{Y_{i}} \frac{\mathrm{d} t}{\bar{G}(t)}}
$$

This choice of $\beta$ reduces the variability. For details, see (11) of Fan and Gijbels [6]. In the estimation procedure, similar to (2.4), we define a theoretic optimal bandwidth $h_{0}^{*}$ which minimizes

$$
J^{*}(h)=\mathbf{E}\left(\hat{g}_{T_{i}^{*}}\left(\theta_{0}^{\tau} X_{i} \mid \theta_{0}\right)-g\left(\theta_{0}^{\tau} X_{i}\right)\right)^{2} I\left(X_{i} \in \mathcal{A}\right),
$$

over all $h$, where

$$
\hat{g}_{T_{i}^{*}}(u \mid \theta)=\frac{\sum_{j \neq i} T_{j}^{*} K_{h}\left(u-\theta^{\tau} X_{j}\right)}{\sum_{j \neq i} K_{h}\left(u-\theta^{\tau} X_{j}\right)}, \quad \text { here } T_{j}^{*}=\delta_{j} \phi_{1}\left(Y_{j}\right)+\left(1-\delta_{j}\right) \phi_{2}\left(C_{j}\right) .
$$


Under some regularity conditions, we prove that the chosen $\tilde{h}_{n}$ is the asymptotically equivalent optimal bandwidth, that is, $\tilde{h}_{n} h_{0}^{*-1} \rightarrow 1$ in probability as $n \rightarrow \infty$.

Define

$$
V^{*}=\int \sigma^{* * 2}(x) \vec{h}_{\mathcal{A}}(x) \vec{h}_{\mathcal{A}}(x)^{\tau} f(x) \mathrm{d} x-\int \frac{\vec{\psi}_{\mathcal{A}_{1}}(s) \vec{\psi}_{\mathcal{A}_{1}}(s)^{\tau}}{\bar{F}(s) \bar{G}^{2}(s)} \mathrm{d} G(s)+V_{\beta}^{*},
$$

where

$$
\begin{aligned}
\sigma^{* * 2}(x)= & \mathbf{E}\left(\left(\varepsilon^{* *}\right)^{2} \mid X=x\right) \\
\vec{\psi}_{\mathcal{A}_{1}}(s)= & \mathbf{E}\left(\delta \phi_{1}(Y) I(Y \geq s) \vec{h}_{\mathcal{A}}(X)\right)+\mathbf{E}\left((1-\delta) \phi_{2}(C) I(C \geq s) \vec{h}_{\mathcal{A}}(X)\right), \\
V_{\beta}^{*}= & (1+\beta)^{2} \int \vec{\psi}_{\mathcal{A}_{2}}(s) \vec{\psi}_{\mathcal{A}_{2}}^{\tau}(s)\left(\int_{0}^{s} \frac{\mathrm{d} t}{\bar{G}(t)}\right)^{2} \frac{\mathrm{d} G(s)}{\bar{F}(s) \bar{G}^{2}(s)} \\
& +(1+\beta) \mathbf{E}\left(\frac{1-\delta}{\bar{F}(C) \bar{G}(C)}\left(\vec{\psi}_{\mathcal{A}_{1}}(C) \oplus \vec{h}_{\mathcal{A}}(X)\right) \int_{0}^{C} \frac{\mathrm{d} t}{\bar{G}(t)}\right) \\
& -(1+\beta)^{2} \mathbf{E}\left(\frac{1-\delta}{\bar{F}(C) \bar{G}(C)}\left(\vec{\psi}_{\mathcal{A}_{1}}(C) \oplus \vec{h}_{\mathcal{A}}(X)\right)\left(\int_{0}^{C} \frac{\mathrm{d} t}{\bar{G}(t)}\right)^{2}\right)
\end{aligned}
$$

$\vec{h}_{\mathcal{A}}(x)$ is given in (3.4), $\phi_{1}(x)$ and $\phi_{2}(x)$ are given in $(1.3), \vec{\psi}_{\mathcal{A}_{2}}(s)=\mathbf{E}(\delta I(Y \geq$ $\left.s) \vec{h}_{\mathcal{A}}(X)\right)+\mathbf{E}\left((1-\delta) I(C \geq s) \vec{h}_{\mathcal{A}}(X)\right)$ and $A \oplus B=A^{\tau} B+B^{\tau} A$. From Fan and Gijbels [6], we see that $\sigma^{* * 2}(x)=\sigma^{2}(x)+d(x)$ where

$$
d(x)=\int_{0}^{+\infty}\left(2 \int_{0}^{y} \int_{0}^{v} \frac{G(s)}{\bar{G}(s)} \mathrm{d} s \mathrm{~d} v+\int_{0}^{y}\left\{\frac{2 \beta y(y-v)-\beta^{2} y^{2}}{\bar{G}^{2}(v)}\right\} \mathrm{d} \bar{G}(v)\right) \mathrm{d} F(y \mid x) .
$$

We now present the main results of this section.

Theorem 4.1 Under conditions $\mathcal{L}$, we have

$n^{\frac{1}{2}}\left(\tilde{\theta}_{n}-\theta_{0}\right) \rightarrow N\left(0, W^{-} V^{*} W^{-}\right) \quad$ in distribution, and $\frac{\tilde{h}_{n}}{h_{0}^{*}} \rightarrow 1 \quad$ in probability,

where $V^{*}$ is given in (4.7) and $W^{-}$denotes the generalized inverse of $W$ in (3.2).

Remark 4.2 If $\beta=-1$, then $\sigma^{* * 2}(x)=\sigma^{* 2}(x), V^{*}=V$ and $W^{-} V^{*} W^{-}=$ $W^{-} V W^{-}$given in Theorem 3.1. If there is no censoring $(G(x) \equiv 0)$, then $\sigma^{* * 2}(x)=\sigma^{2}(x), V^{*}=V_{0}$ and $W^{-} V^{*} W^{-}=W^{-} V_{0} W^{-}$, where $V_{0}$ is given in Remark 3.2. 
Theorem 4.2 Under conditions $\mathcal{L}$ except $(L 8)$, if $\boldsymbol{E}\left(\left(1+|\varepsilon|^{3}\right) \bar{G}^{-3}(Y)\right)<\infty$, we have $\sup _{u \in\left\{\theta^{\tau} x \mid(x, \theta) \in \mathcal{A}^{\delta} \times \Theta_{n}\right\}}\left|\hat{g}_{\hat{T}_{i}^{*}}\left(u \mid \tilde{\theta}_{n}\right)-g(u)\right|=O_{p}\left(n^{-\frac{2}{5}}(\ln n)^{\frac{1}{2}}\right)$, where $\hat{g}_{\hat{T}_{i}^{*}}\left(u \mid \tilde{\theta}_{n}\right)$ and $g(u)$ are defined in (4.4) and (1.1), respectively.

\section{Discussions}

In this paper, we investigate the asymptotic normality of the estimator of the index and the weak convergence rate of the estimator of the link function. It certainly is of interest to study the almost sure convergence and law of iterated logarithm (LIL). Under stronger conditions, most of the arguments used for proving the weak convergence can be employed to prove the strong convergence. For example, if we strengthen condition (L6) in $\mathcal{L}$ with the following two conditions labeled by $\mathcal{H}$ :

(H1) If $b_{F}<b_{G}$, then $\int_{-\infty}^{b_{F}} \bar{F}^{-1} \mathrm{~d} G<\infty$;

(H2) If $b_{F}=b_{G}$, then $\bar{G}^{a} \leq k \bar{F}$ where $k>0$ and $a \in[0,1)$ are constants;

which ensures the LIL for the Kaplan-Meier estimator $G_{n}$ of $G$ under $b_{F} \leq b_{G}$. See Chen and Lo [3] and Gu and Lai [9].

Then, we may obtain the convergence rate of the LIL for $\hat{\theta}_{n}$ as well as the almost-sure optimal convergence rate of the estimator of $g$. However, to prove these, all of the lemmas may need different conditions and some more arguments are needed. Thus, we do not pursue these results in this paper. The relevant study should result in another research paper.

\section{Appendices}

This part consists of four Appendices which present the proofs of all the results given in this paper. Since we use the four lemmas (Lemmas D.1, D.1.1, D.2, D.3 and D.4) in Appendix D of Wang et al. [28] quite frequently in the following appendices, we label them as Lemmas D.1-WHZY05, D.1.1-WHZY05, D.2-WHZY05, D.3-WHZY05, and D.4-WHZY05. The proofs of Theorems 3.1 and 3.2 are included in Appendix A. Since arguments similar to those in the proofs of Theorems 3.1 and 3.2 can be used to prove Theorems 4.1 and 4.2, the proofs of Theorems 4.1 and 4.2 are omitted.

\section{Appendix A}

Recall $S_{n}(\theta, h)$ of (2.2) and rewrite it as

$$
S_{n}(\theta, h)=\sum_{i \in \Lambda_{A}}\left(\hat{T}_{i}-\hat{g}_{\hat{T}_{i}}\left(\theta^{\tau} X_{i} \mid \theta\right)\right)^{2}
$$


Define

$$
\begin{aligned}
t_{i} & =\hat{T}_{i}-T_{i}, \\
\hat{g}_{t_{i}}(\theta, h) & =\hat{g}_{\hat{T}_{i}}\left(\theta^{\tau} X_{i} \mid \theta\right)-\hat{g}_{T_{i}}\left(\theta^{\tau} X_{i} \mid \theta\right), \\
D_{i}(h) & =\hat{g}_{T_{i}}\left(\theta_{0}^{\tau} X_{i} \mid \theta_{0}\right)-g\left(\theta_{0}^{\tau} X_{i}\right), \\
\Delta_{i}(\theta, h) & =\left(\hat{g}_{T_{i}}\left(\theta^{\tau} X_{i} \mid \theta\right)-g\left(\theta^{\tau} X_{i} \mid \theta\right)\right)-D_{i}(h), \\
d_{i}(\theta) & =g\left(\theta^{\tau} X_{i} \mid \theta\right)-g\left(\theta_{0}^{\tau} X_{i}\right), \\
\bar{d}_{i}(\theta) & =\left(\theta-\theta_{0}\right)^{\tau}\left(X_{i}-\vec{\mu}\left(\theta_{0}^{\tau} X_{i}\right)\right) g^{\prime}\left(\theta_{0}^{\tau} X_{i}\right) .
\end{aligned}
$$

After some tedious calculations, we get

$$
S_{n}(\theta, h)=\tilde{S}_{n}(\theta)+J_{n}(h)+R_{1 n}(\theta, h)+R_{2 n}(h)+R_{3 n},
$$

where

$$
\begin{aligned}
\tilde{S}_{n}(\theta)= & \sum_{i \in \Lambda_{A}} \bar{d}_{i}^{2}(\theta)-2 \sum_{i \in \Lambda_{A}} \bar{d}_{i}(\theta)\left(t_{i}+\varepsilon_{i}^{*}\right), \\
J_{n}(h)= & \sum_{i \in \Lambda_{A}} D_{i}^{2}(h)=\sum_{i \in \Lambda_{A}}\left(\hat{g}_{T_{i}}\left(\theta_{0}^{\tau} X_{i} \mid \theta_{0}\right)-g\left(\theta_{0}^{\tau} X_{i}\right)\right)^{2}, \\
R_{1 n}(\theta, h)= & -2\left(\sum_{i \in \Lambda_{A}} \Delta_{i}(\theta, h) \varepsilon_{i}^{*}+\sum_{i \in \Lambda_{A}}\left(d_{i}(\theta)-\bar{d}_{i}(\theta)\right) \varepsilon_{i}^{*}\right)+2 \sum_{i \in \Lambda_{A}} D_{i}(h) d_{i}(\theta) \\
& +2 \sum_{i \in \Lambda_{A}} \hat{g}_{t_{i}}(\theta, h) d_{i}(\theta)-2 \sum_{i \in \Lambda_{A}} \varepsilon_{i}^{*}\left(\hat{g}_{t_{i}}(\theta, h)-\hat{g}_{t_{i}}\left(\theta_{0}, h\right)\right)+\sum_{i \in \Lambda_{A}} \Delta_{i}^{2}(\theta, h) \\
& +2 \sum_{i \in \Lambda_{A}} \Delta_{i}(\theta, h)\left(D_{i}(h)+d_{i}(\theta)-t_{i}+\hat{g}_{t_{i}}(\theta, h)\right) \\
& +\sum_{i \in \Lambda_{A}}\left(\hat{g}_{t_{i}}(\theta, h)^{2}-\hat{g}_{t_{i}}\left(\theta_{0}, h\right)^{2}\right) \\
& +2 \sum_{i \in \Lambda_{A}}\left(D_{i}(h)-t_{i}\right)\left(\hat{g}_{t_{i}}(\theta, h)-\hat{g}_{t_{i}}\left(\theta_{0}, h\right)\right) \\
& +\sum_{i \in \Lambda_{A}}\left(d_{i}(\theta)+\bar{d}_{i}(\theta)-2 t_{i}\right)\left(d_{i}(\theta)-\bar{d}_{i}(\theta)\right), \\
& +\sum_{i \in \Lambda_{A}}\left(2 D_{i}(h)-2 t_{i}+\hat{g}_{t_{i}}\left(\theta_{0}, h\right)\right) \hat{g}_{t_{i}}\left(\theta_{0}, h\right), \\
& \sum_{i \in \Lambda_{A}}\left(t_{i}+\varepsilon_{i}^{*}\right)^{2} . \\
R_{2 n}(h)= & \sum_{i \in \Lambda_{A}}(h) \varepsilon_{i}^{*}-2 \sum_{i \in \Lambda_{A}} D_{i}(h) t_{i}-2 \hat{g}_{t_{i}}\left(\theta_{0}, h\right) \\
& \\
& \\
& \\
&
\end{aligned}
$$


For proving Theorems 3.1 and 3.2, we need the following four lemmas. Note that the proofs of these lemmas require the results presented in Appendices B, C and $\mathrm{D}$. In the proofs, it is assumed that $\xi$ is a positive constant and $0<\alpha<20^{-1}$.

Lemma A.1 Under $\mathcal{L}$, there exists $\lambda>0$, such that

$$
\sup _{(\theta, h) \in \Theta_{n} \times \mathcal{H}_{n}}\left|R_{1 n}(\theta, h)\right|=o_{p}\left(n^{-\lambda}\right) .
$$

Proof of Lemma A.1 From Lemmas B.1-B.4, it can be shown that

$$
\begin{aligned}
\sup _{(\theta, h) \in \Theta_{n} \times \mathcal{H}_{n}}\left|\sum_{i \in \Lambda_{A}} \Delta_{i}(\theta, h) \varepsilon_{i}^{*}+\sum_{i \in \Lambda_{A}}\left(d_{i}(\theta)-\bar{d}_{i}(\theta)\right) \varepsilon_{i}^{*}\right| & =o_{p}\left(n^{-\frac{1}{10}+\alpha+\xi}\right), \\
\sup _{(\theta, h) \in \Theta_{n} \times \mathcal{H}_{n}}\left|\sum_{i \in \Lambda_{A}}\left(D_{i}(h)+\hat{g}_{t_{i}}(\theta, h)\right) d_{i}(\theta)\right| & =O_{p}\left(n^{-\frac{1}{10}+\alpha}(\ln n)^{\frac{1}{2}}\right), \\
\sup _{(\theta, h) \in \Theta_{n} \times \mathcal{H}_{n}}\left|\sum_{i \in \Lambda_{A}}\left(\hat{g}_{t_{i}}(\theta, h)-\hat{g}_{t_{i}}\left(\theta_{0}, h\right)\right) \varepsilon_{i}^{*}\right| & =o_{p}\left(n^{-\frac{1}{10}+2 \alpha}\right) .
\end{aligned}
$$

Moreover, by Lemma C.1(5) and Lemmas C.4-C.6, we get $\sup _{(\theta, h) \in \Theta_{n} \times \mathcal{H}_{n}} \sum_{i \in \Lambda_{A}}$ $\Delta_{i}(\theta, h)^{2}=o_{p}\left(n^{-\frac{2}{5}+2 \alpha+2 \xi}\right)$,

$$
\begin{aligned}
\sup _{(\theta, h) \in \Theta_{n} \times \mathcal{H}_{n}}\left|\sum_{i \in \Lambda_{A}} \Delta_{i}(\theta, h)\left(D_{i}(h)+d_{i}(\theta)-t_{i}+\hat{g}_{t_{i}}(\theta, h)\right)\right| & =o_{p}\left(n^{-\frac{1}{10}+\alpha+\xi}\right), \\
\sup _{(\theta, h) \in \Theta_{n} \times \mathcal{H}_{n}}\left|\sum_{i \in \Lambda_{A}}\left(\hat{g}_{t_{i}}(\theta, h)^{2}-\hat{g}_{t_{i}}\left(\theta_{0}, h\right)^{2}\right)\right| & =O_{p}\left(n^{-\frac{3}{10}+\alpha}\right), \\
\sup _{(\theta, h) \in \Theta_{n} \times \mathcal{H}_{n}}\left|\sum_{i \in \Lambda_{A}}\left(D_{i}(h)-t_{i}\right)\left(\hat{g}_{t_{i}}(\theta, h)-\hat{g}_{t_{i}}\left(\theta_{0}, h\right)\right)\right| & =O_{p}\left(n^{-\frac{1}{5}+\alpha}(\ln n)^{\frac{1}{2}}\right), \\
\sup _{\theta \in \Theta_{n}}\left|\sum_{i \in \Lambda_{A}}\left(d_{i}(\theta)+\bar{d}_{i}(\theta)-2 t_{i}\right)\left(d_{i}(\theta)-\bar{d}_{i}(\theta)\right)\right| & =O_{p}\left(n^{-\frac{1}{2}+3 \alpha}\right) .
\end{aligned}
$$

From the definition of $R_{1 n}(\theta, h)$, we immediately obtain the almost-sure convergence in Lemma A.1.

Lemma A.2 Under $\mathcal{L}$, there exists $0<\omega<10^{-1}$, such that

$$
\sup _{h \in \mathcal{H}_{n}}\left|R_{2 n}(h)\right|=o_{p}\left(n^{\frac{1}{5}-\omega}\right) .
$$


Proof of Lemma A.2 By Lemma B.5, we have $\sup _{h \in \mathcal{H}_{n}}\left|\sum_{i \in \Lambda_{A}} D_{i}(h) \varepsilon_{i}^{*}\right|=O_{p}$ $\left(n^{\frac{1}{10}+2 \xi}\right)$. Furthermore, by Lemmas C.2(1), C.4(2) and C.6, we obtain the convergence rate $\sup _{h \in \mathcal{H}_{n}}\left|\sum_{i \in \Lambda_{A}} t_{i} D_{i}(h)\right|=O_{p}\left(n^{\frac{1}{10}}(\ln n)^{\frac{1}{2}}\right)$,

$$
\sup _{h \in \mathcal{H}_{n}}\left|\sum_{i \in \Lambda_{A}}\left(2 D_{i}(h)-2 t_{i}+\hat{g}_{t_{i}}\left(\theta_{0}, h\right)\right) \hat{g}_{t_{i}}\left(\theta_{0}, h\right)\right|=O_{p}\left(n^{\frac{1}{10}}(\ln n)^{\frac{1}{2}}\right)
$$

Finally, from Lemma B.4, we have $\sup _{h \in \mathcal{H}_{n}}\left|\sum_{i \in \Lambda_{A}} \hat{g}_{t_{i}}\left(\theta_{0}, h\right) \varepsilon_{i}^{*}\right|=O_{p}\left(n^{\frac{1}{10}}(\ln n)^{\frac{1}{2}}\right)$. Hence, the almost-sure convergence in Lemma A.2 holds.

Define

$$
\begin{aligned}
W_{n} & =\frac{1}{n} \sum_{i \in \Lambda_{A}}\left(X_{i}-\vec{\mu}\left(\theta_{0}^{\tau} X_{i}\right)\right)\left(X_{i}-\vec{\mu}\left(\theta_{0}^{\tau} X_{i}\right)\right)^{\tau} g^{\prime}\left(\theta_{0}^{\tau} X_{i}\right)^{2}, \\
Z_{n} & =\frac{1}{n} \sum_{i \in \Lambda_{A}}\left(\hat{T}_{i}-g\left(\theta_{0}^{\tau} X_{i}\right)\right)\left(X_{i}-\vec{\mu}\left(\theta_{0}^{\tau} X_{i}\right)\right) g^{\prime}\left(\theta_{0}^{\tau} X_{i}\right),
\end{aligned}
$$

\section{Lemma A.3 Under $\mathcal{L}$, we have}

$$
\tilde{S}_{n}(\theta)=n\left(\theta-\theta_{0}\right)^{\tau} W_{n}\left(\theta-\theta_{0}\right)-2 n\left(\theta-\theta_{0}\right)^{\tau} Z_{n},
$$

Furthermore, $n^{\frac{1}{2}} Z_{n} \rightarrow N(0, V)$ in distribution, where $V$ is given in (3.3).

Proof of Lemma A.3 The decomposition of $\tilde{S}_{n}(\theta)$

$$
\tilde{S}_{n}(\theta)=n\left(\theta-\theta_{0}\right)^{\tau} W_{n}\left(\theta-\theta_{0}\right)-2 n\left(\theta-\theta_{0}\right)^{\tau} Z_{n}
$$

can be seen from the fact that $\tilde{S}_{n}(\theta)=\sum_{i \in \Lambda_{A}} \bar{d}_{i}^{2}(\theta)-2 \sum_{i \in \Lambda_{A}} \bar{d}_{i}(\theta)\left(\varepsilon_{i}^{*}+t_{i}\right)$, where

$$
\sum_{i \in \Lambda_{A}} \bar{d}_{i}^{2}(\theta)=n\left(\theta-\theta_{0}\right)^{\tau} W_{n}\left(\theta-\theta_{0}\right) \quad \text { and } \quad \sum_{i \in \Lambda_{A}} \bar{d}_{i}(\theta)\left(\varepsilon_{i}^{*}+t_{i}\right)=n\left(\theta-\theta_{0}\right)^{\tau} Z_{n}
$$

Furthermore, using $\vec{h}_{\mathcal{A}}$ of (3.4), we get $Z_{n}=\frac{1}{n} \sum_{i=1}^{n}\left(\frac{\delta_{i} U_{i}}{\vec{G}_{n}\left(U_{i}\right)}-g\left(\theta_{0}^{\tau} X_{i}\right)\right) \vec{h}_{\mathcal{A}}\left(X_{i}\right)$. Finally, using Lemma D.2, one can show that $n^{\frac{1}{2}} Z_{n} \rightarrow N(0, V)$ in distribution. 
Define

$$
\begin{aligned}
K_{1} & =\int u^{2} K(u) \mathrm{d} u, \quad K_{2}=\int K^{2}(u) \mathrm{d} u, \\
A_{1} & =K_{2} \int_{\mathcal{A}} \frac{\sigma_{0}^{2}\left(\theta_{0}^{\tau} x\right)}{v\left(\theta_{0}^{\tau} x\right)} f(x) \mathrm{d} x, \\
A_{2} & =K_{1}^{2} \int_{\mathcal{A}}\left(\frac{g^{\prime \prime}\left(\theta_{0}^{\tau} x\right)}{2}+\frac{g^{\prime}\left(\theta_{0}^{\tau} x\right) v^{\prime}\left(\theta_{0}^{\tau} x\right)}{v\left(\theta_{0}^{\tau} x\right)}\right)^{2} f(x) \mathrm{d} x, \\
\sigma_{0}^{2}(t) & =\mathbf{E}\left(\frac{G(Y-)}{\bar{G}(Y-)} Y^{2} \mid \theta_{0}^{\tau} X=t\right)+\mathbf{E}\left(\varepsilon^{2} \mid \theta_{0}^{\tau} X=t\right),
\end{aligned}
$$

where $v$ is the density function of $\theta_{0}^{\tau} X, v^{\prime}$ is the first derivative of $v$, and $g^{\prime}$ and $g^{\prime \prime}$ are the first and second derivatives of $g$.

Lemma A.4 Under $\mathcal{L}$, we have

$J_{n}(h)=A_{1} h^{-1}+A_{2} n h^{4}+R_{4 n}(h)$ and $J(h)=A_{1}(n h)^{-1}+A_{2} h^{4}+n^{-1} R_{5 n}(h)$,

where $J_{n}(h)$ and $J(h)$ are given in (A.3) and (2.4), respectively. Furthermore,

$$
\sup _{h \in \mathcal{H}_{n}}\left|R_{4 n}(h)\right|=\sup _{h \in \mathcal{H}_{n}}\left|R_{5 n}(h)\right|=o_{p}\left(n^{\frac{1}{5}}\right) .
$$

Proof of Lemma A.4 Under conditions $\mathcal{L}$, we can know that $\mathbf{E}\left(\left|\varepsilon^{*}\right|^{m}\right)<\infty$ for some $m>0$, and $\mathbf{E}\left(\left|\varepsilon^{*}\right|^{2} \mid X=t\right)$ is bounded. Define

$$
\begin{aligned}
C_{n i}\left(\theta_{0}, h\right) & =\frac{1}{n h} \sum_{j \neq i} K_{h}\left(\theta_{0}^{\tau}\left(X_{i}-X_{j}\right)\right), \\
A_{n i}^{\varepsilon}(h) & =\frac{1}{n h} \sum_{j \neq i} \varepsilon_{j}^{*} K_{h}\left(\theta_{0}^{\tau}\left(X_{i}-X_{j}\right)\right), \\
b_{n i}\left(\theta_{0}, h\right) & =\frac{1}{n h} \sum_{j \neq i}\left(g\left(\theta_{0}^{\tau} X_{j}\right)-g\left(\theta_{0}^{\tau} X_{i}\right)\right) K_{h}\left(\theta_{0}^{\tau}\left(X_{i}-X_{j}\right)\right) .
\end{aligned}
$$

Then, from Lemmas D.1-WHZY05, C.3(1) and C.1(2), it follows that

$$
J_{n}(h)=\sum_{i \in \Lambda_{A}}\left(\frac{A_{n i}^{\varepsilon}(h)+b_{n i}\left(\theta_{0}, h\right)}{v\left(\theta_{0}^{\tau} X_{i}\right)}\right)^{2}+R_{1 n}^{J}(h) \equiv \tilde{J}_{n}(h)+R_{1 n}^{J}(h),
$$


where $\sup _{h \in \mathcal{H}_{n}}\left|R_{1 n}^{J}(h)\right|=o_{p}\left(n^{-\frac{3}{5}+4 \xi}\right)$. Write $\tilde{J}_{n}(h)$ as

$$
\begin{aligned}
\tilde{J}_{n}(h) & =\sum_{i \in \Lambda_{A}}\left(\frac{A_{n i}^{\varepsilon}(h)}{v\left(\theta_{0}^{\tau} X_{i}\right)}\right)^{2}+2 \sum_{i \in \Lambda_{A}} \frac{A_{n i}^{\varepsilon}(h) b_{n i}\left(\theta_{0}, h\right)}{v^{2}\left(\theta_{0}^{\tau} X_{i}\right)}+\sum_{i \in \Lambda_{A}} \frac{b_{n i}^{2}\left(\theta_{0}, h\right)}{v^{2}\left(\theta_{0}^{\tau} X_{i}\right)} \\
& \equiv \tilde{J}_{1 n}+\tilde{J}_{2 n}+\tilde{J}_{3 n} .
\end{aligned}
$$

For $\tilde{J}_{2 n}$, we follow the proof of Lemma B.5 in Appendix B to obtain

$$
\sup _{h \in \mathcal{H}_{n}}\left|\tilde{J}_{2 n}\right|=o_{p}\left(n^{\frac{1}{10}+2 \xi}\right) \text {. }
$$

As for $\tilde{J}_{1 n}$, we express it as a sum of two terms

$$
\begin{aligned}
\tilde{J}_{1 n}= & \sum_{i \in \Lambda_{A}} \frac{1}{(n h)^{2}} \sum_{j \neq i}\left(\frac{\varepsilon_{j}^{*} K_{h}\left(\theta_{0}^{\tau}\left(X_{i}-X_{j}\right)\right)}{v\left(\theta_{0}^{\tau} X_{i}\right)}\right)^{2} \\
& +\sum_{i \in \Lambda_{A}} A_{n i}^{\varepsilon}(h) \frac{1}{n h} \sum_{k \neq i, j} \frac{\varepsilon_{k}^{*} K_{h}\left(\theta_{0}^{\tau}\left(X_{i}-X_{k}\right)\right)}{v^{2}\left(\theta_{0}^{\tau} X_{i}\right)} \equiv \tilde{J}_{11 n}+\tilde{J}_{12 n} .
\end{aligned}
$$

Parallel to the derivation of $(A .8)$, we have $\sup _{h \in \mathcal{H}_{n}}\left|\tilde{J}_{12 n}\right|=o_{p}\left(n^{\frac{1}{10}+2 \xi}\right)$. Moreover, by interchanging the order of the two summations in $\tilde{J}_{11 n}$, one can show $\tilde{J}_{11 n}=A_{1} h^{-1}+R_{3 n}^{J}(h)$ where $\sup _{h \in \mathcal{H}_{n}}\left|R_{3 n}^{J}(h)\right|=o_{p}\left(n^{\frac{1}{5}}\right)$ and $A_{1}$ is given in (A.6). Hence, we get

$$
\tilde{J}_{1 n}=\frac{A_{1}}{h}+R_{4 n}^{J}(h)
$$

where $\sup _{h \in \mathcal{H}_{n}}\left|R_{4 n}^{J}(h)\right|=o_{p}\left(n^{\frac{1}{5}}\right)$.

Lastly, for $\tilde{J}_{3 n}$, we let $\Delta b_{n i}=b_{n i}\left(\theta_{0}, h\right)-\mathbf{E}\left(b_{n i}\left(\theta_{0}, h\right) \mid X_{i}\right)$ and split it into three terms

$\tilde{J}_{3 n}=\sum_{i \in \Lambda_{A}}\left(\frac{\Delta b_{n i}}{v\left(\theta_{0}^{\tau} X_{i}\right)}\right)^{2}+2 \sum_{i \in \Lambda_{A}} \frac{\Delta b_{n i} \mathbf{E}\left(b_{n i}\left(\theta_{0}, h\right) \mid X_{i}\right)}{v^{2}\left(\theta_{0}^{\tau} X_{i}\right)}+\sum_{i \in \Lambda_{A}}\left(\frac{\mathbf{E}\left(b_{n i}\left(\theta_{0}, h\right) \mid X_{i}\right)}{v\left(\theta_{0}^{\tau} X_{i}\right)}\right)^{2}$.

Mimicking the derivation of (A.9) and using Lemma C.3(3), we have

$$
\tilde{J}_{3 n}=A_{2} n h^{4}+R_{5 n}^{J}(h)
$$

where $\sup _{h \in \mathcal{H}_{n}}\left|R_{5 n}^{J}(h)\right|=o_{p}\left(n^{\frac{1}{5}}\right)$ and $A_{2}$ is given in (A.7). Therefore, by (A.8), (A.9) and (A.10), it can be easily shown that $J_{n}(h)=A_{1} h^{-1}+A_{2} n h^{4}+R_{4 n}(h)$, where $\sup _{h \in \mathcal{H}_{n}}\left|R_{4 n}(h)\right|=o_{p}\left(n^{\frac{1}{5}}\right)$. Note that the notation $R_{4 n}(h)$ is used in the lemma. 
Repeating the steps of the above proof for $J_{n}(h)$, it can be shown that $n J(h)=$ $A_{1} h^{-1}+A_{2} n h^{4}+R_{5 n}(h)$, where $\sup _{h \in \mathcal{H}_{n}}\left|R_{5 n}(h)\right|=o_{p}\left(n^{\frac{1}{5}}\right)$. Also, the notation $R_{5 n}(h)$ is used in the lemma.

We now turn to the proofs of Theorems 3.1 and 3.2.

Proof of Theorem 3.1 Applying Lemma A.3, we have

$$
\begin{aligned}
S_{n}(\theta, h)= & n\left(\theta-\theta_{0}-W_{n}^{-} Z_{n}\right)^{\tau} W_{n}\left(\theta-\theta_{0}-W_{n}^{-} Z_{n}\right)-n Z_{n}^{\tau} W_{n}^{-} Z_{n} \\
& +J_{n}(h)+R_{1 n}(\theta, h)+R_{2 n}(h)+R_{3 n} .
\end{aligned}
$$

Let $\theta_{n}^{*}=\theta_{0}+W_{n}^{-} Z_{n}$. Then, Lemma A.3 together with the fact that $W_{n}^{-}=$ $W^{-}+o_{p}(1)$ yield $\theta_{n}^{*}-\theta_{0}=O_{p}\left(n^{-\frac{1}{2}}\right)$. Hence, $\theta_{n}^{*} \in \Theta_{n}$ in probability. Since $\hat{\theta}_{n}$ and $\hat{h}_{n}$ minimize $S_{n}(\theta, h), S_{n}\left(\hat{\theta}_{n}, \hat{h}_{n}\right) \leq S_{n}\left(\theta_{n}^{*}, \hat{h}_{n}\right)$. That is,

$$
n\left(\hat{\theta}_{n}-\theta_{0}-W_{n}^{-} Z_{n}\right)^{\tau} W_{n}\left(\hat{\theta}_{n}-\theta_{0}-W_{n}^{-} Z_{n}\right)+R_{1 n}\left(\hat{\theta}_{n}, \hat{h}_{n}\right) \leq R_{1 n}\left(\theta_{n}^{*}, \hat{h}_{n}\right) .
$$

Hence, from Lemma A.1, we have $n\left(\hat{\theta}_{n}-\theta_{0}-W_{n}^{-} Z_{n}\right)^{\tau} W_{n}\left(\hat{\theta}_{n}-\theta_{0}-W_{n}^{-} Z_{n}\right)=$ $o_{p}\left(n^{-\lambda}\right)$, where $\lambda$ is a positive constant. Furthermore, we get $W_{n}^{\frac{1}{2}}\left(\hat{\theta}_{n}-\theta_{0}-\right.$ $\left.W_{n}^{-} Z_{n}\right)=o_{p}\left(n^{-\frac{1+\lambda}{2}}\right)$, or equivalently,

$$
W_{n}\left(\hat{\theta}_{n}-\theta_{0}\right)=Z_{n}+o_{p}\left(n^{-\frac{1+\lambda}{2}}\right) .
$$

From Lemma 1 of Xia et al. [29], we have $\operatorname{rank}(W)=p-1$ and $\operatorname{rank}\left(W_{n}\right)=$ $p-1$ for large $n$. Recall that $\left\|\hat{\theta}_{n}\right\|_{2}=1$ and that the first component of $\hat{\theta}_{n}$ is nonnegative. Therefore, $\hat{\theta}_{n}$ is a unique solution of (A.11). Thus, $\hat{\theta}_{n}-\theta_{0}=$ $W_{n}^{-} Z_{n}+o_{p}\left(n^{-\frac{1+\lambda}{2}}\right)$. Again from Lemma A.3, we have $\lim _{\sup _{n \rightarrow \infty}} n^{\frac{1}{2}}\left(\hat{\theta}_{n}-\right.$ $\left.\theta_{0}\right) \rightarrow N\left(0, W^{-} V W^{-}\right)$in distribution.

Let $R_{1 n}^{*}(\theta)=\tilde{S}_{n}(\theta)+R_{3 n}$. Then, it follows from Lemma A.1, A.2 and A.4 that $S_{n}\left(\hat{\theta}_{n}, h\right)-R_{1 n}^{*}\left(\hat{\theta}_{n}\right)=A_{1} h^{-1}+A_{2} n h^{4}+o_{p}\left(n^{\frac{1}{5}}\right)$, uniformly in $\mathcal{H}_{n}$. Since $\hat{h}_{n}$ minimizes $S_{n}\left(\hat{\theta}_{n}, h\right)$, we obtain from Lemma A.4 that $\hat{h}_{n} h_{0}^{-1} \rightarrow 1$ in probability.

Proof of Theorem 3.2 Let $I_{n}=\sup _{u \in\left\{\theta^{\tau} x \mid(x, \theta) \in \mathcal{A}^{\delta} \times \Theta_{n}\right\}}\left|\hat{g}_{\hat{T}_{i}}\left(u \mid \hat{\theta}_{n}\right)-g(u)\right|$ where $\hat{g}_{\hat{T}_{i}}(u \mid \theta)$ is defined in Sect. 2. Then,

$$
I_{n} \leq \sup _{(x, \theta, h) \in \Omega_{n}}\left|\hat{g}_{\hat{T}_{i}}\left(\theta^{\tau} x \mid \theta\right)-g\left(\theta_{0}^{\tau} x\right)\right|+\sup _{(x, \theta, h) \in \Omega_{n}}\left|g\left(\theta_{0}^{\tau} x\right)-g\left(\theta^{\tau} x\right)\right| .
$$

Denote $J_{n}^{*}=\hat{g}_{\hat{T}_{i}}\left(\theta^{\tau} x \mid \theta\right)-g\left(\theta_{0}^{\tau} x\right)$. Therefore,

$$
J_{n}^{*}=\frac{g_{n i}^{t}(x, \theta, h)+A_{n i}^{\varepsilon}(x, \theta, h)+\hat{b}_{n i}(x, \theta, h)}{C_{n i}(x, \theta, h)},
$$


where

$$
\begin{aligned}
& g_{n i}^{t}(x, \theta, h)=\frac{1}{(n-1) h} \sum_{j \neq i} t_{j} K_{h}\left(\theta^{\tau}\left(x-X_{j}\right)\right), \\
& A_{n i}^{\varepsilon}(x, \theta, h)=\frac{1}{(n-1) h} \sum_{j \neq i} \varepsilon_{j}^{*} K_{h}\left(\theta^{\tau}\left(x-X_{j}\right)\right), \\
& \hat{b}_{n i}(x, \theta, h)=\frac{1}{(n-1) h} \sum_{j \neq i}\left(g\left(\theta_{0}^{\tau} X_{j}\right)-g\left(\theta_{0}^{\tau} X_{i}\right)\right) K_{h}\left(\theta^{\tau}\left(x-X_{j}\right)\right), \\
& C_{n i}(x, \theta, h)=\frac{1}{(n-1) h} \sum_{j \neq i} K_{h}\left(\theta^{\tau}\left(x-X_{j}\right)\right) .
\end{aligned}
$$

As $\mathbf{E}\left(\left(1+|\varepsilon|^{3}\right) \bar{G}^{-3}(Y)\right)<\infty$, it is easy to see that $\mathbf{E}\left(\left(1+|\varepsilon|^{2}\right) \bar{G}^{-3}(Y)\right)<\infty$ and $\mathbf{E}\left(\left|\varepsilon^{*}\right|^{3}\right)<\infty$. And condition (L7) in $\mathcal{L}$ implies that $\mathbf{E}\left(\left|\varepsilon^{*}\right|^{2} \mid X=t\right)$ is bounded. Hence, we obtain $\sup _{(x, \theta, h) \in \Omega_{n}}\left|J_{n}^{*}\right|=O_{p}\left(n^{-\frac{2}{5}}(\ln n)^{\frac{1}{2}}\right)$ by applying (C.7) in Appendix C to $g_{n i}^{t}(x, \theta, h)$, Lemma D.1-WHZY05 to $A_{n i}^{\varepsilon}(x, \theta, h)$, Lemma C.3(2) to $\hat{b}_{n i}(x, \theta, h)$, and Lemma C.1 (2) to $C_{n i}^{-1}(x, \theta, h)$. It is clear that $\sup _{(x, \theta, h) \in \Omega_{n}}\left|g\left(\theta_{0}^{\tau} x\right)-g\left(\theta^{\tau} x\right)\right|=O_{p}\left(n^{-\frac{1}{2}+\alpha}\right)$. This together with (A.12) and (A.13) yield $\left|I_{n}\right|=O_{p}\left(n^{-\frac{2}{5}}(\ln n)^{\frac{1}{2}}\right)$.

\section{Appendix B}

The proofs for the lemmas are basically the outlines. The readers are referred to Wang et al. [28] for details.

Define monotone increasing $\sigma$-fields

$$
\mathbf{F}_{n 0}=\mathbf{F}_{X}=\sigma\left(X_{i}, 1 \leq i \leq n\right) \text { and } \mathbf{F}_{n, i}=\mathbf{F}_{X} \vee \sigma\left(\varepsilon_{1}^{*}, \varepsilon_{2}^{*}, \ldots, \varepsilon_{i}^{*}\right), 1 \leq i \leq n,
$$

where $\varepsilon_{\mathbf{i}}^{*}$ is given in (1.2).

Lemma B.1 Under $\mathcal{L}$, we have

$$
\sup _{(\theta, h) \in \Theta_{n} \times \mathcal{H}_{n}}\left|\sum_{i \in \Lambda_{A}} \Delta_{i} \varepsilon_{i}^{*}+\sum_{i \in \Lambda_{A}}\left(d_{i}-\bar{d}_{i}\right) \varepsilon_{i}^{*}\right|=o_{p}\left(n^{-\frac{1}{10}+\alpha+\xi}\right),
$$

where $\Delta_{i} \equiv \Delta_{i}(\theta, h)$, di $\equiv d_{i}(\theta), \bar{d}_{i} \equiv \bar{d}_{i}(\theta)$ are given in (A.1), and $\varepsilon_{i}^{*}$ is given in (1.2).

Proof of Lemma B.1 Let $\varphi_{n}(\theta, h)=\sum_{i \in \Lambda_{A}} \Delta_{i} \varepsilon_{i}^{*}+\sum_{i \in \Lambda_{A}}\left(d_{i}-\bar{d}_{i}\right) \varepsilon_{i}^{*}$. We first prove that for all $l \geq 1$,

$$
\sup _{(\theta, h) \in \Theta_{n} \times \mathcal{H}_{n}} \mathbf{E}\left(\left|\varphi_{n}(\theta, h)\right|^{2 l}\right)=O\left(n^{-\frac{1}{5} l+2 \alpha l}\right) .
$$


Divide $\varphi_{n}(\theta, h)$ into two parts

$$
\begin{aligned}
\varphi_{n}(\theta, h) & =\sum_{i \in \Lambda_{A}}\left(\mathbf{E}\left(\Delta_{i} \mid \mathbf{F}_{X}\right)+\left(d_{i}-\bar{d}_{i}\right)\right) \varepsilon_{i}^{*}+\sum_{i \in \Lambda_{A}}\left(\Delta_{i}-\mathbf{E}\left(\Delta_{i} \mid \mathbf{F}_{X}\right)\right) \varepsilon_{i}^{*} \\
& \equiv \varphi_{n 1}(\theta, h)+\varphi_{n 2}(\theta, h) .
\end{aligned}
$$

By Minkovski's inequality, we have

$$
\mathbf{E}\left(\left|\varphi_{n}(\theta, h)\right|^{2 l}\right) \leq 2^{2 l-1}\left(\mathbf{E}\left(\left|\varphi_{n 1}(\theta, h)\right|^{2 l}\right)+\mathbf{E}\left(\left|\varphi_{n 2}(\theta, h)\right|^{2 l}\right)\right) .
$$

By Burkholder's inequality and Hölder's inequality, the dominated convergence theorem and Lemma C.5 in Appendix C, (C.14) of Appendix C, and $\mathbf{E}\left(\left|\varepsilon_{i}^{*}\right|^{2 l m_{1}^{\prime}}\right)<\infty$ from $(\mathrm{L} 8)$ in $\mathcal{L}$,

$$
\sup _{(\theta, h) \in \Theta_{n} \times \mathcal{H}_{n}} \mathbf{E}\left(\left|\varphi_{n 1}(\theta, h)\right|^{2 l}\right)=o\left(n^{-\frac{2}{5} l+2 \alpha l+2 \xi l}\right) .
$$

Similarly, we can derive that $\sup _{(\theta, h) \in \Theta_{n} \times \mathcal{H}_{n}} \mathbf{E}\left(\left|\varphi_{n 2}(\theta, h)\right|^{2 l}\right)=O\left(n^{-\frac{1}{5} l+2 \alpha l}\right)$, we can show that (B.2) holds.

To complete the proof of the lemma, we can prove that for all $\left(\theta_{1}, h_{1}\right),\left(\theta_{2}, h_{2}\right) \in$ $\Theta_{n} \times \mathcal{H}_{n}$, there exists $L^{\prime}>0$ such that

$$
\mathbf{E}\left(\left|\varphi_{n}\left(\theta_{1}, h_{1}\right)-\varphi_{n}\left(\theta_{2}, h_{2}\right)\right|\right) \leq L^{\prime} n^{2}\left(\left\|\theta_{1}-\theta_{2}\right\|_{2}+\left|h_{1}-h_{2}\right|\right) .
$$

Let $\delta^{\prime}=n^{-\frac{21}{10}}\left(2 L^{\prime}\right)^{-1}$. Then, we can find a dense discrete subset $\Theta_{n}^{\prime} \times \mathcal{H}_{n}^{\prime} \in \Theta_{n} \times$ $\mathcal{H}_{n}$, whose cardinality $N=\#\left(\mathcal{H}_{n}^{\prime}\right) \leq n^{2 p}$, satisfies that for all $(\theta, h) \in \Theta_{n} \times \mathcal{H}_{n}$, there exists $\left(\theta^{\prime}, h^{\prime}\right) \in \Theta_{n}^{\prime} \times \mathcal{H}_{n}^{\prime}$ such that $\left\|\theta-\theta^{\prime}\right\|_{2} \leq \delta^{\prime}$ and $\left|h-h^{\prime}\right| \leq \delta^{\prime}$.

Note that for any $\epsilon>0$,

$P\left(\sup _{(\theta, h) \in \Theta_{n}^{\prime} \times \mathcal{H}_{n}^{\prime}}\left|\varphi_{n}(\theta, h)\right|>\epsilon n^{-\frac{1}{10}+\alpha+\xi}\right) \leq \sum_{k=1}^{N} \frac{\mathbf{E}\left(\left|\varphi_{n}\left(\theta_{k}, h_{k}\right)\right|^{2 l}\right)}{\epsilon^{2 l} n^{-\frac{1}{5} l+2 l \alpha+2 l \xi}}=o\left(n^{2 p-2 l \xi}\right)$,

where the last inequality is due to (B.2). Then, for any $\xi>0$, putting $l>$ $(p+1) \xi^{-1}$, we obtain $\sup _{(\theta, h) \in \Theta_{n}^{\prime} \times \mathcal{H}_{n}^{\prime}}\left|\varphi_{n}(\theta, h)\right|=o_{p}\left(n^{-\frac{1}{10}+\alpha+\xi}\right)$. Again from (B.5), we get $\sup _{(\theta, h) \in \Theta_{n} \times \mathcal{H}_{n}}\left|\varphi_{n}(\theta, h)\right|=o_{p}\left(n^{-\frac{1}{10}+\alpha+\xi}\right)$. Hence, the lemma holds.

Lemma B.2 Under $\mathcal{L}$, we have

$$
\sup _{(\theta, h) \in \Theta_{n} \times \mathcal{H}_{n}}\left|\sum_{i \in \Lambda_{A}} D_{i}(h) d_{i}(\theta)\right|=O_{p}\left(n^{-\frac{1}{10}+\alpha}(\ln n)^{\frac{1}{2}}\right),
$$

where $D_{i}(h)$ and $d_{i}(\theta)$ are given in (A.1). 
Proof of Lemma B.2

$$
\begin{gathered}
\sum_{i \in \Lambda_{A}} D_{i}(h) d_{i}(\theta)=\sum_{i \in \Lambda_{A}} \mathbf{E}\left(D_{i}(h) \mid \mathbf{F}_{X}\right) \bar{d}_{i}(\theta)+\sum_{i \in \Lambda_{A}}\left(D_{i}(h)-\mathbf{E}\left(D_{i}(h) \mid \mathbf{F}_{X}\right)\right) \bar{d}_{i}(\theta) \\
+\sum_{i \in \Lambda_{A}} D_{i}(h)\left(d_{i}(\theta)-\bar{d}_{i}(\theta)\right) \equiv I_{1}+I_{2}+I_{3}
\end{gathered}
$$

From Lemma C.4(2) and Lemma C.5, one can easily get

$$
\sup _{(\theta, h) \in \Theta_{n} \times \mathcal{H}_{n}}\left|I_{3}\right|=O_{p}\left(n^{-\frac{2}{5}+2 \alpha}(\ln n)^{\frac{1}{2}}\right) .
$$

By Lemma D.1-WHZY05, Lemma C.1(2) and Lemma C.5, we have

$$
\sup _{(\theta, h) \in \Theta_{n} \times \mathcal{H}_{n}}\left|I_{1}\right|=O_{p}\left(n^{-\frac{1}{10}+\alpha}(\ln n)^{\frac{1}{2}}\right) .
$$

Invoking Lemmas D.1-WHZY05, C.1(2) and C.5 and Using the same arguments as in the proof of Lemma B.1, we easily get $\sup _{(\theta, h) \in \Theta_{n} \times \mathcal{H}_{n}}\left|I_{2}\right|=O_{p}$ $\left(n^{-\frac{3}{10}+\alpha} \ln n\right)$. This in conjunction with (B.6), (B.7) and (B.8) give the lemma.

Lemma B.3 Under $\mathcal{L}$ except $(L 8)$, if $\boldsymbol{E}\left(\left(1+|\varepsilon|^{2}\right) \bar{G}^{-3}(Y)\right)<\infty$, we have

$$
\sup _{(\theta, h) \in \Theta_{n} \times \mathcal{H}_{n}}\left|\sum_{i \in \Lambda_{A}} \hat{g}_{t_{i}}(\theta, h) d_{i}(\theta)\right|=O_{p}\left(n^{-\frac{3}{10}+2 \alpha}\right),
$$

where $\hat{g}_{t_{i}}(\theta, h)$ and $d_{i}(\theta)$ are given in (A.1).

Proof of Lemma B.3 Express $\sum_{i \in \Lambda_{A}} \hat{g}_{t_{i}}(\theta, h) d_{i}(\theta)$ as

$$
\begin{aligned}
\sum_{i \in \Lambda_{A}} \hat{g}_{t_{i}}(\theta, h) d_{i}(\theta)= & \sum_{i \in \Lambda_{A}}\left(\hat{g}_{t_{i}}(\theta, h)-\hat{g}_{t_{i}}\left(\theta_{0}, h\right)\right) d_{i}(\theta) \\
& +\sum_{i \in \Lambda_{A}} \hat{g}_{t_{i}}\left(\theta_{0}, h\right)\left(d_{i}(\theta)-\bar{d}_{i}(\theta)\right) \\
& +\sum_{i \in \Lambda_{A}} \hat{g}_{t_{i}}\left(\theta_{0}, h\right) \bar{d}_{i}(\theta) \equiv I_{1}+I_{2}+I_{3} .
\end{aligned}
$$

It is easy to see from Lemma C.2(1), Lemma C.2(2) and Lemma C.5 that

$$
\sup _{(\theta, h) \in \Theta_{n} \times \mathcal{H}_{n}}\left|I_{1}+I_{2}\right|=O_{p}\left(n^{-\frac{3}{10}+2 \alpha}\right) .
$$

Using $r_{t_{i}}^{(1)}$ in Lemma C.2(3), and Lemma D.1-WHZY05, we can get $\sup _{(x, h) \in \mathcal{C} \times \mathcal{H}_{n}}\left|f^{(1)}(x, h)\right|=O_{p}\left(n^{-\frac{2}{5}}(\ln n)^{\frac{1}{2}}\right)$. This together with Lemmas C.2(3), C.5 
and C.6 give $\sup _{(\theta, h) \in \Theta_{n} \times \mathcal{H}_{n}}\left|I_{3}\right|=O_{p}\left(n^{-\frac{2}{5}+\alpha}(\ln n)^{\frac{1}{2}}\right)$. Hence, again from (B.9) and (B.10), we get the lemma.

Lemma B.4 Under $\mathcal{L}$ except (L8), if $\boldsymbol{E}\left|\varepsilon^{*}\right|^{3}<\infty$, we have

(1) $\sup _{(\theta, h) \in \Theta_{n} \times \mathcal{H}_{n}}\left|\sum_{i \in \Lambda_{A}}\left(\hat{g}_{t_{i}}(\theta, h)-\hat{g}_{t_{i}}\left(\theta_{0}, h\right)\right) \varepsilon_{i}^{*}\right|=o_{p}\left(n^{-\frac{1}{10}+2 \alpha}\right)$,

(2) $\sup _{h \in \mathcal{H}_{n}}\left|\sum_{i \in \Lambda_{A}} \hat{g}_{t_{i}}\left(\theta_{0}, h\right) \varepsilon_{i}^{*}\right|=O_{p}\left(n^{\frac{1}{10}}(\ln n)^{\frac{1}{2}}\right)$.

Proof of Lemma B.4 We only prove (1). By Lemma C.2(4),

$$
\begin{aligned}
\sum_{i \in \Lambda_{A}}\left(\hat{g}_{t_{i}}(\theta, h)-\hat{g}_{t_{i}}\left(\theta_{0}, h\right)\right) \varepsilon_{i}^{*} & \\
= & \sum_{i \in \Lambda_{A}}\left(K_{i}^{t}(\theta, h)+r_{t_{i}}^{(2)}(\theta, h)\right) \varepsilon_{i}^{*} \\
= & \sum_{i \in \Lambda_{A}} \varepsilon_{i}^{*}\left(\frac{1}{n h} \sum_{j=1}^{n} t_{j} \frac{K_{h}\left(\theta^{\tau}\left(X_{i}-X_{j}\right)\right)-K_{h}\left(\theta_{0}^{\tau}\left(X_{i}-X_{j}\right)\right)}{v\left(\theta_{0}^{\tau} X_{i}\right)}\right) \\
& -\sum_{i \in \Lambda_{A}} \varepsilon_{i}^{*}\left(\frac{1}{n h} \sum_{j=1}^{n} t_{j} K_{h}\left(\theta_{0}^{\tau}\left(X_{i}-X_{j}\right)\right)\left(\left(\theta-\theta_{0}\right)^{\tau} \frac{\vec{\phi}_{1}\left(X_{i}\right)}{v^{2}\left(\theta_{0}^{\tau} X_{i}\right)}\right)\right) \\
& +\sum_{i \in \Lambda_{A}} \varepsilon_{i}^{*} \frac{K(0)}{n h} t_{i}\left(\theta-\theta_{0}\right)^{\tau} \frac{\vec{\phi}_{1}\left(X_{i}\right)}{v^{2}\left(\theta_{0}^{\tau} X_{i}\right)}+\sum_{i \in \Lambda_{A}} \varepsilon_{i}^{*} r_{t_{i}}^{(2)}(\theta, h) \\
\equiv & I_{1}+\left(\theta-\theta_{0}\right)^{\tau} I_{2}+I_{3}+I_{4} .
\end{aligned}
$$

where $K_{i}^{t}(\theta, h)$ is given in (C.6) and $\vec{\phi}_{1}(x)$ is given in Lemma C.1.

Interchanging the two summations in $I_{1}$ and $I_{2}$ respectively, we have

$$
I_{1}=\sum_{j=1}^{n} t_{j}\left(f^{(2)}\left(X_{j}, \theta, h\right)-f^{(2)}\left(X_{j}, \theta_{0}, h\right)\right) \quad \text { and } \quad I_{2}=-\sum_{j=1}^{n} \vec{t}_{j}^{(3)}\left(X_{j}, h\right)
$$

where

$$
\begin{aligned}
f^{(2)}(x, \theta, h) & =\frac{1}{n h} \sum_{i=1}^{n} \frac{\varepsilon_{i}^{*}}{v\left(\theta_{0}^{\tau} X_{i}\right)} I\left(X_{i} \in \mathcal{A}\right) K_{h}\left(\theta^{\tau}\left(x-X_{i}\right)\right), \\
\vec{f}^{(3)}(x, h) & =\frac{1}{n h} \sum_{i=1}^{n} \frac{\varepsilon_{i}^{*} \vec{\phi}_{1}\left(X_{i}\right)}{v^{2}\left(\theta_{0}^{\tau} X_{i}\right)} I\left(X_{i} \in \mathcal{A}\right) K_{h}\left(\theta_{0}^{\tau}\left(x-X_{i}\right)\right) .
\end{aligned}
$$

Since $\mathbf{E}\left(\left|\varepsilon^{*}\right|^{3}\right)<\infty$ and $\mathbf{E}\left(\left|\varepsilon_{i}^{*}\right|^{2} \mid X=t\right)$ is bounded from condition (L7) in $\mathcal{L}$, Lemmas D.1-WHZY05, D.2-WHZY05 and C.6 yield $\sup _{(\theta, h) \in \Theta_{n} \times \mathcal{H}_{n}}\left|I_{1}\right|=$ $o_{p}\left(n^{-\frac{1}{5}+\xi+\alpha}\right)$ and $\sup _{(\theta, h) \in \Theta_{n} \times \mathcal{H}_{n}}\left|I_{2}\right|=O_{p}\left(n^{\frac{1}{10}}(\ln n)^{\frac{1}{2}}\right)$, Lemma C.4(3) and C.6 
yield $\sup _{(\theta, h) \in \Theta_{n} \times \mathcal{H}_{n}}\left|I_{3}\right|=O_{p}\left(n^{-\frac{2}{5}+\alpha}\right)$, and Lemma C.2(4)implies $\sup _{(\theta, h) \in \Theta_{n} \times \mathcal{H}_{n}}$ $\left|I_{4}\right|=o_{p}\left(n^{-\frac{1}{10}+2 \alpha}\right)$. Hence, $(1)$ in the lemma holds.

Lemma B.5 Under $\mathcal{L}$, we have

$$
\sup _{h \in \mathcal{H}_{n}}\left|\sum_{i \in \Lambda_{A}} D_{i}(h) \varepsilon_{i}^{*}\right|=o_{p}\left(n^{\frac{1}{10}+2 \xi}\right) .
$$

Proof of Lemma B.5 Applying the same argument as in the proof of Lemma B.1, we get

$$
\sup _{h \in \mathcal{H}_{n}} \mathbf{E}\left(\left|\sum_{i \in \Lambda_{A}} D_{i}(h) \varepsilon_{i}^{*}\right|^{2 l}\right)=o\left(n^{\frac{1}{5} l+2 l \xi}\right),
$$

for all $l \geq 1$. We can find a dense subset $\mathcal{H}_{n}^{\prime} \in \mathcal{H}_{n}$ so that $\sup _{h \in \mathcal{H}_{n}^{\prime}}\left|\sum_{i \in \Lambda_{A}} D_{i}(h) \varepsilon_{i}^{*}\right|$ $=o_{p}\left(n^{\frac{1}{10}+2 \xi}\right)$ and using the uniform continuity of $\sum_{i \in \Lambda_{A}} D_{i}(h) \varepsilon_{i}^{*}$ with respect to $h$, we finally obtain $\sup _{h \in \mathcal{H}_{n}}\left|\sum_{i \in \Lambda_{A}} D_{i}(h) \varepsilon_{i}^{*}\right|=o_{p}\left(n^{\frac{1}{10}+2 \xi}\right)$.

\section{Appendix C}

Recall the density function $v$ of $\theta_{0}^{\tau} X$ and $\Omega_{n}, \bar{\Omega}_{n}$ and $\Lambda_{A}$ defined in Sect. 3. The following lemma gives the uniform convergence rate of the kernel estimator of $v$.

Lemma C.1 Let $C_{n}(x, \theta, h)=(n h)^{-1} \sum_{j=1}^{n} K_{h}\left(\theta^{\tau}\left(x-X_{j}\right)\right)$. Under the regularity conditions (L1)-(L4) in $\mathcal{L}$, we get

(1) $C_{n}(x, \theta, h)=v\left(\theta_{0}^{\tau} x\right)+r_{C}^{(1)}(x, \theta, h)$, with $\sup _{(x, \theta, h) \in \Omega_{n}}\left|r_{C}^{(1)}(x, \theta, h)\right|=O_{p}\left(n^{-\frac{2}{5}}(\ln n)^{\frac{1}{2}}\right)$;

(2) $C_{n}^{-1}(x, \theta, h)=v\left(\theta_{0}^{\tau} x\right)^{-1}+r_{C}^{(2)}(x, \theta, h)$, with $\sup _{(x, \theta, h) \in \Omega_{n}}\left|r_{C}^{(2)}(x, \theta, h)\right|=O_{p}\left(n^{-\frac{2}{5}}(\ln n)^{\frac{1}{2}}\right)$; $C_{n}(x, \theta, h)-C_{n}\left(x, \theta_{0}, h\right)=\left(\theta-\theta_{0}\right)^{\tau} \vec{\phi}_{1}(x)+r_{C}^{(3)}(x, \theta, h)$, with $\sup _{(x, \theta, h) \in \Omega_{n}}\left|r_{C}^{(3)}(x, \theta, h)\right|=o_{p}\left(n^{-\frac{3}{5}+2 \alpha}\right)$, for $0<\alpha<\frac{1}{10}$; $C_{n}^{-1}(x, \theta, h)-C_{n}^{-1}\left(x, \theta_{0}, h\right)=-\left(\theta-\theta_{0}\right)^{\tau} \vec{\phi}_{1}(x) v^{-2}\left(\theta_{0}^{\tau} x\right)+r_{C}^{(4)}(x, \theta, h)$, with $\sup _{(x, \theta, h) \in \Omega_{n}}\left|r_{C}^{(4)}(x, \theta, h)\right|=o_{p}\left(n^{-\frac{3}{5}+2 \alpha}\right)$, for $0<\alpha<\frac{1}{10}$.

Where $\vec{\phi}_{1}(x)=\left(x-\vec{\mu}\left(\theta_{0}^{\tau} x\right)\right) v^{\prime}\left(\theta_{0}^{\tau} x\right)+\vec{\mu}^{\prime}\left(\theta_{0}^{\tau} x\right) v\left(\theta_{0}^{\tau} x\right)$, here $\vec{\mu}$ is given in (3.1), and $\nu^{\prime}$ and $\vec{\mu}^{\prime}$ are respectively the first derivatives of $\nu$ and $\vec{\mu}$. 
Proof of Lemma C.1 We prove the lemma in the following order: (3) $\Rightarrow$ (1) $\Rightarrow$ (2) $\Rightarrow$ (4).

Let $I_{n}=C_{n}(x, \theta, h)-C_{n}\left(x, \theta_{0}, h\right)$ and $\tilde{K}_{j}(x, \theta, h)=K_{h}\left(\theta^{\tau}\left(x-X_{j}\right)\right)-$ $K_{h}\left(\theta_{0}^{\tau}\left(x-X_{j}\right)\right)$. So, $\mathbf{E}\left(I_{n}\right)=h^{-1} \mathbf{E}\left(\tilde{K}_{j}(x, \theta, h)\right)$. Then, Taylor's expansion yields

$$
\mathbf{E}\left(I_{n}\right)=\frac{1}{h^{2}}\left(\theta-\theta_{0}\right)^{\tau} \mathbf{E}\left(K_{h}^{\prime}\left(\theta_{0}^{\tau}\left(x-X_{j}\right)\right)\left(x-X_{j}\right)\right)+R_{1 n}^{I}(x, \theta, h),
$$

where $\left|R_{1 n}^{I}(x, \theta, h)\right| \leq L_{1}\left\|\theta-\theta_{0}\right\|_{2}^{2} h^{-3} P\left(\left|\frac{\theta_{0}^{\tau}\left(x-X_{j}\right)}{h}\right| \leq 2\right)=L_{2}\left\|\theta-\theta_{0}\right\|_{2}^{2} h^{-2}$, where $L_{1}$ and $L_{2}$ are positive constants. Since (L4) in $\mathcal{L}$ implies $\int_{-1}^{1} K^{\prime}(u) \mathrm{d} u=0$, $\int_{-1}^{1} u K^{\prime}(u) \mathrm{d} u=-1$ and $\int_{-1}^{1} u^{2} K^{\prime}(u) \mathrm{d} u=0$, applying Taylor's expansion to $\vec{\mu}(\cdot)$ and $\nu(\cdot)$ about $\theta_{0}^{\tau} x$ gives

$$
\begin{aligned}
\mathbf{E}\left(K_{h}^{\prime}\left(\theta_{0}^{\tau}\left(x-X_{j}\right)\right)\left(x-X_{j}\right)\right) & =\mathbf{E}\left(\left(x-\vec{\mu}\left(\theta_{0}^{\tau} X_{j}\right)\right) K_{h}^{\prime}\left(\theta_{0}^{\tau}\left(x-X_{j}\right)\right)\right) \\
& =h \int_{-1}^{1}\left(x-\vec{\mu}\left(\theta_{0}^{\tau} x-h u\right)\right) v\left(\theta_{0}^{\tau} x-h u\right) K^{\prime}(u) \mathrm{d} u \\
& =h^{2} \vec{\phi}_{1}(x)+R_{2 n}^{I}(x, h)
\end{aligned}
$$

It is easy to see that $R_{2 n}^{I}(x, h) \leq L_{3} h^{3}$ where $L_{3}$ is a positive constant.

From (C.1) and (C.2), it follows that $\mathbf{E}\left(I_{n}\right)=\left(\theta-\theta_{0}\right)^{\tau} \vec{\phi}_{1}(x)+R_{3 n}^{I}(x, \theta, h)$, with $\sup _{(x, \theta, h) \in \Omega_{n}}\left|R_{3 n}^{I}(x, \theta, h)\right|=O\left(n^{-\frac{3}{5}+2 \alpha}\right)$. Thus, we obtain $\sup _{(x, \theta, h) \in \Omega_{n}}\left|\mathbf{E}\left(I_{n}\right)\right|=$ $O\left(n^{-\frac{1}{2}+\alpha}\right), \sup _{(x, \theta, h) \in \Omega_{n}}\left|\mathbf{E}\left(I_{n}\right)-\left(\theta-\theta_{0}\right)^{\tau} \vec{\phi}_{1}(x)\right|=O\left(n^{-\frac{3}{5}+2 \alpha}\right)$. These together with Lemma D.2-WHZY05 lead to

$$
\sup _{(x, \theta, h) \in \Omega_{n}}\left|I_{n}\right|=O_{p}\left(n^{-\frac{1}{2}+\alpha}\right),
$$

$$
\sup _{(x, \theta, h) \in \Omega_{n}}\left|I_{n}-\left(\theta-\theta_{0}\right)^{\tau} \vec{\phi}_{1}(x)\right|=O_{p}\left(n^{-\frac{3}{5}+2 \alpha}\right) .
$$

Note that (C.4) implies (3) of the lemma. Furthermore, (C.3), Lemmas D.1WHZY05 and D.3-WHZY05 imply (1) which results in (2). Finally, we get (4) from (2) and (C.4).

Note that $t_{i}$ and $\hat{g}_{t_{i}}(\theta, h)$ of (A.1) can be written as

$$
t_{i}=\left(\frac{1}{\bar{G}_{n}\left(Y_{i}\right)}-\frac{1}{\bar{G}\left(Y_{i}\right)}\right) \delta_{i} Y_{i} \quad \text { and } \quad \hat{g}_{t_{i}}(\theta, h)=\frac{g_{n i}^{t}(\theta, h)}{C_{n i}(\theta, h)}
$$


where

$$
\begin{aligned}
& g_{n i}^{t}(\theta, h)=(n h)^{-1} \sum_{j \neq i} t_{j} K_{h}\left(\theta^{\tau}\left(X_{i}-X_{j}\right)\right), \\
& C_{n i}(\theta, h)=(n h)^{-1} \sum_{j \neq i} K_{h}\left(\theta^{\tau}\left(X_{i}-X_{j}\right)\right) .
\end{aligned}
$$

Define

$$
K_{i}^{t}(\theta, h)=\frac{g_{n i}^{t}(\theta, h)-g_{n i}^{t}\left(\theta_{0}, h\right)}{v\left(\theta_{0}^{\tau} X_{i}\right)}-\left(\theta-\theta_{0}\right)^{\tau} g_{n i}^{t}\left(\theta_{0}, h\right) \frac{\vec{\phi}_{1}\left(X_{i}\right)}{v^{2}\left(\theta_{0}^{\tau} X_{i}\right)},
$$

where $\vec{\phi}_{1}(x)$ is defined in Lemma C.1.

The following lemma gives a uniform convergence rate of $\hat{g}_{t_{i}}(\theta, h)$, the kernel estimator of $t_{i}$ under random censorship.

Lemma C.2 Under $\mathcal{L}$ except $(L 8)$, if $\boldsymbol{E}\left(\left(1+|\varepsilon|^{2}\right) \bar{G}^{-3}(Y)\right)<\infty$, we have

(1) $\sup _{i \in \Lambda_{A}} \sup _{(\theta, h) \in \Theta_{n} \times \mathcal{H}_{n}}\left|\hat{g}_{t_{i}}(\theta, h)\right|=O_{p}\left(n^{-\frac{1}{2}}\right)$;

(2) $\sup _{i \in \Lambda_{A}} \sup _{(\theta, h) \in \Theta_{n} \times \mathcal{H}_{n}}\left|\hat{g}_{t_{i}}(\theta, h)-\hat{g}_{t_{i}}\left(\theta_{0}, h\right)\right|=O_{p}\left(n^{-\frac{8}{10}+\alpha}\right)$;

(3) $\hat{g}_{t_{i}}(\theta, h)=g_{n i}^{t}(\theta, h) v^{-1}\left(\theta_{0}^{\tau} X_{i}\right)+r_{t_{i}}^{(1)}(\theta, h)$, with $\sup _{i \in \Lambda_{A}} \sup _{(\theta, h) \in \Theta_{n} \times \mathcal{H}_{n}}\left|r_{t_{i}}^{(1)}(\theta, h)\right|=O_{p}\left(n^{-\frac{9}{10}}(\ln n)^{\frac{1}{2}}\right)$;

(4) $\hat{g}_{t_{i}}(\theta, h)-\hat{g}_{t_{i}}\left(\theta_{0}, h\right)=K_{i}^{t}(\theta, h)+r_{t_{i}}^{(2)}(\theta, h)$, with $\sup _{i \in \Lambda_{A}} \sup _{(\theta, h) \in \Theta_{n} \times \mathcal{H}_{n}}\left|r_{t_{i}}^{(2)}(\theta, h)\right|=O_{p}\left(n^{-\frac{11}{10}+2 \alpha}\right)$.

Proof of Lemma C.2 Define $g_{n}^{t}(x, \theta, h)=(n h)^{-1} \sum_{j=1}^{n} t_{j} K_{h}\left(\theta^{\tau}\left(x-X_{j}\right)\right)$. We only prove the following two formulas:

$$
\begin{gathered}
\sup _{(x, \theta, h) \in \Omega_{n}}\left|g_{n}^{t}(x, \theta, h)\right|=O_{p}\left(n^{-\frac{1}{2}}\right), \\
\sup _{(x, \theta, h) \in \Omega_{n}}\left|g_{n}^{t}(x, \theta, h)-g_{n}^{t}\left(x, \theta_{0}, h\right)\right|=O_{p}\left(n^{-\frac{8}{10}+\alpha}\right)
\end{gathered}
$$

since Lemma C.2 can be derived from (C.7) and (C.8) and Lemma C.1. Let

$$
\begin{aligned}
A_{n}^{Y}(x, \theta, h) & =\frac{1}{n h} \sum_{j=1}^{n} \frac{\delta_{j}\left|Y_{j}\right|}{\bar{G}^{2}\left(Y_{j}\right)} K_{h}\left(\theta^{\tau}\left(x-X_{j}\right)\right), \\
\tilde{A}_{n}^{Y}(x, h) & =\frac{1}{n h} \sum_{j=1}^{n} \frac{\delta_{j}\left|Y_{j}\right|}{\bar{G}^{2}\left(Y_{j}\right)}\left|K_{h}^{\prime}\left(\theta_{0}^{\tau}\left(x-X_{j}\right)\right)\right| .
\end{aligned}
$$


By $\mathcal{L}$, Lemma D.1.1-WHZY05,

$$
\sup _{(x, \theta, h) \in \Omega_{n}} A_{n}^{Y}(x, \theta, h)=O_{p}(1), \quad \text { and } \quad \sup _{(x, h) \in \mathcal{A}^{\delta} \times \mathcal{H}_{n}} \tilde{A}_{n}^{Y}(x, h)=O_{p}(1) \text {. (C.9) }
$$

Thus, by (C.9) and Lemma C.7, we have

$$
\left|g_{n}^{t}(x, \theta, h)\right| \leq \sup _{y \leq U_{n, n}}\left|R_{n}^{G}(y)\right| A_{n}^{Y}(x, \theta, h)=O_{p}\left(n^{-\frac{1}{2}}\right)
$$

where $\sup _{y \leq U_{n, n}}\left|R_{n}^{G}(y)\right|=O_{p}\left(n^{-\frac{1}{2}}\right)$. Hence, (C.7) holds. Invoking Lemmas C.6 and C.7., (C.8) can be similarly proved.

Define

$$
\begin{aligned}
& b_{n}(x, \theta, h)=\frac{1}{n h} \sum_{j=1}^{n}\left(g\left(\theta^{\tau} X_{j}\right)-g\left(\theta^{\tau} x\right)\right) K_{h}\left(\theta^{\tau}\left(x-X_{j}\right)\right), \\
& \hat{b}_{n}(x, \theta, h)=\frac{1}{n h} \sum_{j=1}^{n}\left(g\left(\theta_{0}^{\tau} X_{j}\right)-g\left(\theta_{0}^{\tau} x\right)\right) K_{h}\left(\theta^{\tau}\left(x-X_{j}\right)\right) .
\end{aligned}
$$

Lemma C.3 Under conditions (L1)-(L4) in $\mathcal{L}$, we have

(1) $\sup _{(x, \theta, h) \in \Omega_{n}}\left|b_{n}(x, \theta, h)\right|=O_{p}\left(n^{-\frac{2}{5}}\right)$;

(2) $\sup _{(x, \theta, h) \in \Omega_{n}}\left|\hat{b}_{n}(x, \theta, h)\right|=O_{p}\left(n^{-\frac{2}{5}}\right)$;

(3) $\sup _{(x, \theta, h) \in \Omega_{n}}\left|b_{n}(x, \theta, h)-\boldsymbol{E}\left(b_{n}(x, \theta, h)\right)\right|=O_{p}\left(n^{-\frac{3}{5}}(\ln n)^{\frac{1}{2}}\right)$;

(4) $\sup _{(x, \theta, h) \in \bar{\Omega}_{n}}\left|b_{n}(x, \theta, h)-b_{n}\left(x, \theta_{0}, h\right)\right|=o_{p}\left(n^{-\frac{7}{10}+\alpha+\xi}\right)$.

Proof of Lemma C.3 We prove the lemma in the following order: (4) $\Rightarrow$ (1), (2) and (3). Let $b_{n}^{*}=b_{n}(x, \theta, h)-b_{n}\left(x, \theta_{0}, h\right)$. We first prove

$$
\sup _{(x, \theta, h) \in \bar{\Omega}_{n}}\left|\mathbf{E}\left(b_{n}^{*}\right)\right|=O\left(n^{-\frac{7}{10}+\alpha}\right) .
$$

Since by Taylor's expansion,

$$
\begin{aligned}
\mathbf{E}\left(b_{n}^{*}\right)= & \frac{1}{h} \mathbf{E}\left(\left(\left(g\left(\theta^{\tau} X_{j}\right)-g\left(\theta_{0}^{\tau} X_{j}\right)\right)-\left(g\left(\theta^{\tau} x\right)-g\left(\theta_{0}^{\tau} x\right)\right)\right) K_{h}\left(\theta^{\tau}\left(x-X_{j}\right)\right)\right) \\
& +\frac{1}{h} \mathbf{E}\left(\left(g\left(\theta_{0}^{\tau} X_{j}\right)-g\left(\theta_{0}^{\tau} x\right)\right)\left(K_{h}\left(\theta^{\tau}\left(x-X_{j}\right)\right)-K_{h}\left(\theta_{0}^{\tau}\left(x-X_{j}\right)\right)\right)\right), \\
\equiv & I_{1}+I_{2}+I_{3}+I_{4}+I_{5},
\end{aligned}
$$


where

$$
\begin{aligned}
& I_{1}=\frac{1}{h}\left(\theta-\theta_{0}\right)^{\tau} \mathbf{E}\left(\left(X_{j} g^{\prime}\left(\theta_{0}^{\tau} X_{j}\right)-x g^{\prime}\left(\theta_{0}^{\tau} x\right)\right) K_{h}\left(\theta_{0}^{\tau}\left(x-X_{j}\right)\right)\right), \\
& I_{2}=\frac{1}{h}\left(\theta-\theta_{0}\right)^{\tau} \mathbf{E}\left(\left(X_{j} g^{\prime}\left(\theta_{0}^{\tau} X_{j}\right)-x g^{\prime}\left(\theta_{0}^{\tau} x\right)\right)\left(K_{h}\left(\theta^{\tau}\left(x-X_{j}\right)\right)-K_{h}\left(\theta_{0}^{\tau}\left(x-X_{j}\right)\right)\right)\right), \\
& I_{3}=\frac{1}{2 h} \mathbf{E}\left(g^{\prime \prime}\left(\xi_{1}\right)\left(\left(\theta-\theta_{0}\right)^{\tau} X_{j}\right)^{2} K_{h}\left(\theta^{\tau}\left(x-X_{j}\right)\right)\right), \\
& I_{4}=\frac{1}{h^{2}}\left(\theta-\theta_{0}\right)^{\tau} \mathbf{E}\left(\left(g\left(\theta_{0}^{\tau} X_{j}\right)-g\left(\theta_{0}^{\tau} x\right)\right)\left(x-X_{j}\right) K_{h}^{\prime}\left(\theta_{0}^{\tau}\left(x-X_{j}\right)\right)\right), \\
& I_{5}=\frac{1}{2 h^{3}} \mathbf{E}\left(\left(g\left(\theta_{0}^{\tau} X_{j}\right)-g\left(\theta_{0}^{\tau} x\right)\right)\left(\left(\theta-\theta_{0}\right)^{\tau}\left(x-X_{j}\right)\right)^{2} K_{h}^{\prime \prime}\left(\xi_{2}\right)\right),
\end{aligned}
$$

$\xi_{1}$ is between $\theta^{\tau} X_{j}$ and $\theta_{0}^{\tau} X_{j}$ and $\xi_{2}$ is between $h^{-1} \theta^{\tau}\left(x-X_{j}\right)$ and $h^{-1} \theta_{0}^{\tau}\left(x-X_{j}\right)$. It is easy to see that $\sup _{(x, \theta, h) \in \bar{\Omega}_{n}}\left|I_{2}+I_{3}\right|=O\left(n^{-\frac{4}{5}+2 \alpha}\right)$. Also, $\sup _{(x, \theta, h) \in \bar{\Omega}_{n}}\left|I_{5}\right|=$ $O\left(n^{-\frac{4}{5}+2 \alpha}\right)$ because of

$$
\begin{aligned}
\left|I_{5}\right| & \leq O\left(\frac{\left\|\theta-\theta_{0}\right\|_{2}^{2}}{h^{3}} \mathbf{E}\left(\left|\left(g\left(\theta_{0}^{\tau} X_{j}\right)-g\left(\theta_{0}^{\tau} x\right)\right) K_{h}^{\prime \prime}\left(\xi_{2}\right)\right|\right)\right) \\
& =O\left(\frac{\left\|\theta-\theta_{0}\right\|_{2}^{2}}{h^{3}} h P\left(\left|\theta_{0}^{\tau}\left(X_{j}-x\right)\right|<2 h\right)\right)=O\left(\frac{\left\|\theta-\theta_{0}\right\|_{2}^{2}}{h}\right) .
\end{aligned}
$$

Moreover, $I_{1}+I_{4}=h^{-1}\left(\theta-\theta_{0}\right)^{\tau} I_{6}+h^{-2}\left(\theta-\theta_{0}\right)^{\tau} I_{7}$ where

$$
\begin{aligned}
I_{6} & =\mathbf{E}\left(\left(\vec{\mu}\left(\theta_{0}^{\tau} X_{j}\right) g^{\prime}\left(\theta_{0}^{\tau} X_{j}\right)-x g^{\prime}\left(\theta_{0}^{\tau} x\right)\right) K_{h}\left(\theta_{0}^{\tau}\left(x-X_{j}\right)\right)\right) \\
& =h\left(\vec{\mu}\left(\theta_{0}^{\tau} x\right)-x\right) g^{\prime}\left(\theta_{0}^{\tau} x\right) v\left(\theta_{0}^{\tau} x\right)+O\left(h^{2}\right), \\
I_{7} & =\mathbf{E}\left(\left(g\left(\theta_{0}^{\tau} X_{j}\right)-g\left(\theta_{0}^{\tau} x\right)\right)\left(x-\vec{\mu}\left(\theta_{0}^{\tau} X_{j}\right)\right) K_{h}^{\prime}\left(\theta_{0}^{\tau}\left(x-X_{j}\right)\right)\right) \\
& =h^{2}\left(x-\vec{\mu}\left(\theta_{0}^{\tau} x\right)\right) g^{\prime}\left(\theta_{0}^{\tau} x\right) v\left(\theta_{0}^{\tau} x\right)+O\left(h^{3}\right) .
\end{aligned}
$$

Then, we have $I_{1}+I_{4}=O\left(h\left\|\theta-\theta_{0}\right\|_{2}\right)$. Hence, (C.11) holds.

We next write $b_{n}(x, \theta, h)$ as

$$
b_{n}(x, \theta, h)=\hat{b}_{n}(x, \theta, h)+\left(\theta-\theta_{0}\right)^{\tau} \vec{b}_{2 n}(x, \theta, h)+b_{3 n}(x, \theta, h),
$$

where

$$
\begin{aligned}
\vec{b}_{2 n}(x, \theta, h) & =\frac{1}{n h} \sum_{j=1}^{n}\left(X_{j} g^{\prime}\left(\theta_{0}^{\tau} X_{j}\right)-x g^{\prime}\left(\theta_{0}^{\tau} x\right)\right) K_{h}\left(\theta^{\tau}\left(x-X_{j}\right)\right), \\
\left|b_{3 n}(x, \theta, h)\right| & \leq\left\|\theta-\theta_{0}\right\|_{2}^{2} C_{n}(x, \theta, h) .
\end{aligned}
$$


Applying Lemma D.2-WHZY05 to $\hat{b}_{n}(x, \theta, h)-\hat{b}_{n}\left(x, \theta_{0}, h\right)$ and Lemma D.1WHZY05 to $\vec{b}_{2 n}(x, \theta, h)$, respectively, we obtain

$$
\sup _{(x, \theta, h) \in \bar{\Omega}_{n}}\left|b_{n}^{*}-\mathbf{E}\left(b_{n}^{*}\right)\right|=o_{p}\left(n^{-\frac{7}{10}+\alpha+\xi}\right) .
$$

This together with (C.11) yield (4) of the lemma.

Similarly, by Lemma D.1-WHZY05, Lemma D.3-WHZY05 (4) of the lemma, it is easily seen that (1) and (2) hold and then (3).

Lemma C.4 Under conditions (L1)-(L4) in $\mathcal{L}$, if $\boldsymbol{E}\left(\left|\varepsilon^{*}\right|^{3}\right)<\infty$, then

(1) $\sup _{i \in \Lambda_{A}} \sup _{(\theta, h) \in \Theta_{n} \times \mathcal{H}_{n}}\left|\Delta_{i}(\theta, h)\right|=o_{p}\left(n^{-\frac{7}{10}+\alpha+\xi}\right)$,

(2) $\sup _{i \in \Lambda_{A}} \sup _{h \in \mathcal{H}_{n}}\left|D_{i}(h)\right|=O_{p}\left(n^{-\frac{2}{5}}(\ln n)^{\frac{1}{2}}\right)$,

(3) $\sup _{1 \leq i \leq n}\left|\varepsilon_{i}^{*}\right|=o_{p}\left(n^{\frac{2}{5}}\right)$,

where $\Delta_{i}(\theta, h)$ and $D_{i}(h)$ are given in (A.1), and $\varepsilon_{i}^{*}$ is given in (1.2).

Proof of Lemma C.4 We only prove (1). Note that $\Delta_{i}(\theta, h)$ can be written as

$$
\Delta_{i}(\theta, h)=\mathbf{E}\left(\Delta_{i}(\theta, h) \mid \mathbf{F}_{X}\right)+\left(\Delta_{i}(\theta, h)-\mathbf{E}\left(\Delta_{i}(\theta, h) \mid \mathbf{F}_{X}\right)\right) \equiv I_{1}+I_{2} .
$$

We now prove that for $j=1,2$,

$$
\sup _{i \in \Lambda_{A}} \sup _{(\theta, h) \in \Theta_{n} \times \mathcal{H}_{n}}\left|I_{j}\right|=o_{p}\left(n^{-\frac{7}{10}+\alpha+\xi}\right) .
$$

Let $e_{n i}(\theta, h)=(n h)^{-1} \sum_{j \neq i}\left(g\left(\theta_{0}^{\tau} X_{j}\right)-g\left(\theta^{\tau} X_{i} \mid \theta\right)\right) C_{n i}^{-1}(\theta, h)$. Then, $I_{1}=e_{n i}(\theta, h)$ $-e_{n i}\left(\theta_{0}, h\right)$. Taylor's expansion yields

$$
\begin{aligned}
g\left(\theta_{0}^{\tau} X_{j}\right)-g\left(\theta^{\tau} X_{i} \mid \theta\right)= & \left(\theta-\theta_{0}\right)^{\tau}\left(\vec{\mu}\left(\theta_{0}^{\tau} X_{i}\right) g^{\prime}\left(\theta_{0}^{\tau} X_{i}\right)-X_{j} g^{\prime}\left(\theta_{0}^{\tau} X_{j}\right)\right) \\
& +\left(g\left(\theta^{\tau} X_{j}\right)-g\left(\theta^{\tau} X_{i}\right)\right)-R_{1 n}^{e}\left(\theta, X_{i}, X_{j}\right),
\end{aligned}
$$

with $\sup _{i \in \Lambda_{\mathcal{A}}, j \in \Lambda_{\mathcal{A}}^{\delta}} \sup _{\theta \in \Theta_{n}}\left|R_{1 n}^{e}\left(\theta, X_{i}, X_{j}\right)\right|=O_{p}\left(n^{-1+2 \alpha}\right)$. Hence,

$$
e_{n i}(\theta, h)=a_{n i}(\theta, h)+\tilde{a}_{n i}(\theta, h)+R_{2 n}^{e}\left(X_{i}, \theta, h\right)
$$

where

$$
\begin{aligned}
& a_{n i}(\theta, h)=\frac{b_{n i}(\theta, h)}{C_{n i}(\theta, h)}, \quad \tilde{a}_{n i}(\theta, h)=-\left(\theta-\theta_{0}\right)^{\tau} \frac{\tilde{b}_{n i}(\theta, h)}{C_{n i}(\theta, h)}, \\
& b_{n i}(\theta, h)=\frac{1}{n h} \sum_{j \neq i}\left(g\left(\theta^{\tau} X_{j}\right)-g\left(\theta^{\tau} X_{i}\right)\right) K_{h}\left(\theta^{\tau}\left(X_{i}-X_{j}\right)\right), \\
& \tilde{b}_{n i}(\theta, h)=\frac{1}{n h} \sum_{j \neq i}\left(X_{j} g^{\prime}\left(\theta_{0}^{\tau} X_{j}\right)-\vec{\mu}\left(\theta_{0}^{\tau} X_{i}\right) g^{\prime}\left(\theta_{0}^{\tau} X_{i}\right)\right) K_{h}\left(\theta^{\tau}\left(X_{i}-X_{j}\right)\right),
\end{aligned}
$$


and $\sup _{i \in \Lambda_{A}} \sup _{(\theta, h) \in \Theta_{n} \times \mathcal{H}_{n}}\left|R_{2 n}^{e}\left(X_{i}, \theta, h\right)\right|=O_{p}\left(n^{-1+2 \alpha}\right)$. Furthermore, we have

$$
\begin{aligned}
I_{1}= & \frac{b_{n i}(\theta, h)-b_{n i}\left(\theta_{0}, h\right)}{C_{n i}(\theta, h)}+b_{n i}\left(\theta_{0}, h\right)\left(\frac{1}{C_{n i}(\theta, h)}-\frac{1}{C_{n i}\left(\theta_{0}, h\right)}\right) \\
& +\tilde{a}_{n i}(\theta, h)+R_{3 n}^{e}\left(X_{i}, \theta, h\right),
\end{aligned}
$$

where $\sup _{i \in \Lambda_{A}} \sup _{(\theta, h) \in \Theta_{n} \times \mathcal{H}_{n}}\left|R_{3 n}^{e}\left(X_{i}, \theta, h\right)\right|=O_{p}\left(n^{-1+2 \alpha}\right)$. Since Lemmas D.1WHZY05, D.3-WHZY05 and D.4-WHZY05 imply $\sup _{i \in \Lambda_{A}} \sup _{(\theta, h) \in \Theta_{n} \times \mathcal{H}_{n}}$ $\left\|\tilde{b}_{n i}(\theta, h)\right\|_{2}=O_{p}\left(n^{-\frac{3}{10}+\alpha}\right)$, we use Lemma C.1(2) to get $\sup _{i \in \Lambda_{A}} \sup _{(\theta, h) \in \Theta_{n} \times \mathcal{H}_{n}}$ $\left|\tilde{a}_{n i}(\theta, h)\right|=o_{p}\left(n^{-\frac{7}{10}+\alpha}\right)$. Hence, by (C.16), Lemmas C.1(2), C.1(4), C.3(1), and C.3(4), one can show that (C.14) holds for $j=1$.

As for $I_{2}$, we let $A_{n}^{\varepsilon}(x, \theta, h)=(n h)^{-1} \sum_{j=1}^{n} \varepsilon_{j}^{*} K_{h}\left(\theta^{\tau}\left(x-X_{j}\right)\right)$. Then,

$$
\begin{aligned}
I_{2}= & \frac{A_{n}^{\varepsilon}\left(X_{i}, \theta, h\right)-A_{n}^{\varepsilon}\left(X_{i}, \theta_{0}, h\right)}{C_{n i}(\theta, h)}+A_{n}^{\varepsilon}\left(X_{i}, \theta_{0}, h\right)\left(\frac{1}{C_{n i}(\theta, h)}-\frac{1}{C_{n i}\left(\theta_{0}, h\right)}\right) \\
& -\frac{1}{n h} K(0) \varepsilon_{i}^{*}\left(\frac{1}{C_{n i}(\theta, h)}-\frac{1}{C_{n i}\left(\theta_{0}, h\right)}\right) .
\end{aligned}
$$

Applying Lemma D.1-WHZY05 to $A_{n}^{\varepsilon}(x, \theta, h)$ and Lemma D.2-WHZY05 to $A_{n}^{\varepsilon}(x, \theta, h)-A_{n}^{\varepsilon}\left(x, \theta_{0}, h\right)$, we obtain $\sup _{i \in \Lambda_{A}} \sup _{(\theta, h) \in \Theta_{n} \times \mathcal{H}_{n}}\left|I_{2}\right|=o_{p}\left(n^{-\frac{7}{10}+\alpha+\xi}\right)$ from Lemmas C.1(2), C.1(4), and (3) of the lemma. Hence, (C.14) holds for $j=2$. It completes the proof of (1) of the lemma.

Lemma C.5 Under conditions (L2)-(L3) in $\mathcal{L}$, we have $\sup _{i \in \Lambda_{A}} \sup _{\theta \in \Theta_{n}}\left|d_{i}(\theta)\right|=O_{p}\left(n^{-\frac{1}{2}+\alpha}\right) \quad$ and $\sup _{i \in \Lambda_{A}} \sup _{\theta \in \Theta_{n}}\left|d_{i}(\theta)-\bar{d}_{i}(\theta)\right|=O_{p}\left(n^{-1+2 \alpha}\right)$,

where $d_{i}(\theta)$ and $\bar{d}_{i}(\theta)$ are given in (A.1).

Proof of Lemma C.5 By the definition of $\bar{d}_{i}(\theta)$ and (C.15) with $j=i$, we can prove the lemma holds.

Lemma C.6 Under conditions (L5)-(L7) in $\mathcal{L}$, we have

$$
\sum_{i=1}^{n}\left|t_{i}\right|=O_{p}\left(n^{\frac{1}{2}}\right)
$$

where $t_{i}=\hat{T}_{i}-T_{i}$ is given in (A.1).

Proof of Lemma C.6 It follows from Lemma C.7. 
Lemma C.7 Under conditions (L5)-(L6) in $\mathcal{L}$, we have

$$
\frac{1}{\bar{G}_{n}(y-)}-\frac{1}{\bar{G}(y)}=\frac{R_{n}^{G}(y)}{\bar{G}^{2}(y)},
$$

where $\sup _{y \leq U_{n, n}}\left|R_{n}^{G}(y)\right|=O_{p}\left(n^{-\frac{1}{2}}\right)$, here $U_{n, n}=\max \left\{U_{1}, U_{2}, \ldots, U_{n}\right\}$.

Proof of Lemma C.7 Under conditions (L5)-(L6) in $\mathcal{L}$, we have $\sup _{y \leq U_{n, n}} \sqrt{n} \mid G_{n}$ $(y-)-G(y) \mid=O_{p}(1)$ because $\sqrt{n}\left(G_{n}\left(y \wedge U_{n, n}\right)-G\left(y \wedge U_{n, n}\right)\right)$ is tight in $D[-\infty,+\infty]$.(see [8]).

Let $s_{n}(y)=\left|\bar{G}_{n}(y-)-\bar{G}(y)\right|$. From the fact that

$$
\left|\frac{1}{\bar{G}_{n}(y-)}-\frac{1}{\bar{G}(y)}\right| \leq \frac{s_{n}(y)}{\bar{G}(y)}\left|\frac{1}{\bar{G}_{n}(y-)}-\frac{1}{\bar{G}(y)}\right|+\frac{s_{n}(y)}{\bar{G}^{2}(y)},
$$

we get

$$
\left|\frac{1}{\bar{G}_{n}(y-)}-\frac{1}{\bar{G}(y)}\right| \leq\left(\frac{s_{n}(y)}{\bar{G}_{n}(y-)}+1\right) \frac{s_{n}(y)}{\bar{G}^{2}(y)} .
$$

Let $R_{n}^{G}(y)=\bar{G}^{2}(y)\left(\bar{G}_{n}^{-1}(y-)-\bar{G}^{-1}(y)\right)$. Since $\sup _{y \leq U_{n, n}} s_{n}(y) \bar{G}_{n}^{-1}(y-)=O_{p}(1)$ from Zhou [30] hence, we show that $\sup _{y \leq U_{n, n}}\left|R_{n}^{G}(y)\right|=O_{p}\left(n^{-\frac{1}{2}}\right)$.

\section{Appendix D}

Recall model (1.1). For any bounded and measurable function $h(x)$, we define

$$
Z_{n}=\frac{1}{n} \sum_{i=1}^{n}\left(\frac{\delta_{i} U_{i}}{\bar{G}_{n}\left(U_{i}\right)}-g\left(\theta_{0}^{\tau} X_{i}\right)\right) h\left(X_{i}\right)
$$

In this appendix, we give the asymptotic properties of $Z_{n}$.

Recall that $F(s)=P(Y \leq s), G(s)=P(C \leq s)$, and $F^{0}(x, y)=P(X \leq$ $x, Y \leq y)$ where $s, y \in \mathbb{R}, x \in \mathbb{R}^{p}$. For any two vectors $a=\left(a_{1}, a_{2}, \ldots, a_{p}\right)^{\tau}$ and $b=\left(b_{1}, b_{2}, \ldots, b_{p}\right)^{\tau}$, the inequality $a \leq b$ means that $a_{i} \leq b_{i}$ for $i=1,2, \ldots, p$. Denote by $f(x)$ the density function of $X$.

Define $H^{0}(x)=P(U \leq x, \delta=0)$ and $\bar{H}(x)=1-H(x)=P(U>x)$. Let $F^{*}(x, y)=P(X \leq x, U \leq y, \delta=1)$ be the joint distribution of the random vector $(X, U, \delta=1)$. Then, one can show that

$$
F^{*}(x, y)=\mathbf{E}(P(X \leq x, U \leq y, \delta=1 \mid X, Y))=\iint_{u \leq x} \int_{v \leq y} \bar{G}(v-) F^{0}(\mathrm{~d} u, \mathrm{~d} v) .
$$


It follows immediately that the joint distribution of $(X, Y)$ is given by

$$
F^{0}(x, y)=\int_{u \leq x} \int_{v \leq y} \frac{1}{\bar{G}(v-)} F^{*}(\mathrm{~d} u, \mathrm{~d} v) .
$$

The empirical version of $F^{*}$ is given by

$$
F_{n}^{*}(x, y)=\frac{1}{n} \sum_{j=1}^{n} I\left(X_{j} \leq x, U_{j} \leq y, \delta_{j}=1\right) .
$$

In (D.2), replacing $F^{*}$ and $G$ by the empirical counterpart $F_{n}^{*}$ and the time-honored Kaplan-Meier estimator $G_{n}$ yields an estimator of $F^{0}(x, y)$

$$
F_{n}^{0}(x, y)=\frac{1}{n} \sum_{j=1}^{n} \frac{\delta_{j}}{\bar{G}_{n}\left(U_{j}-\right)} I\left(X_{j} \leq x, U_{j} \leq y\right),
$$

where $\bar{G}_{n}=1-G_{n}$. Let $M_{n}=n^{-1} \sum_{i=1}^{n} g\left(\theta_{0}^{\tau} X_{i}\right) h\left(X_{i}\right)$. Then,

$$
Z_{n}=\int y h(x) F_{n}^{0}(\mathrm{~d} x, \mathrm{~d} y)-M_{n} .
$$

In order to derive the asymptotic properties of $Z_{n}$, let $b<b_{F}$ such that $F(b)>0$. Let $\phi_{b}(x, y)=h(x)$ y $I(y \leq b)$. Define

$$
\begin{aligned}
\mu(b) & =\int \phi_{b}(x, y) F^{0}(\mathrm{~d} x, \mathrm{~d} y), \quad \psi_{b}(s)=\int_{y \geq s} \phi_{b}(x, y) F^{0}(\mathrm{~d} x, \mathrm{~d} y), \\
\alpha(b) & =\int g\left(\theta_{0}^{\tau} x\right) h(x) \phi_{b}(x, y) F^{0}(\mathrm{~d} x, \mathrm{~d} y), \\
\mu_{g} & =\int g\left(\theta_{0}^{\tau} x\right) h(x) f(x) \mathrm{d} x, \quad m_{g}^{2}=\int g^{2}\left(\theta_{0}^{\tau} x\right) h^{2}(x) f(x) \mathrm{d} x,
\end{aligned}
$$

and $Z_{n}(b)=\int \phi_{b}(x, y) F_{n}^{0}(\mathrm{~d} x, \mathrm{~d} y)-M_{n}$. Then, we have

Lemma D.1 Let $F$ and $G$ be continuous and $b<b_{F}$ such that $F(b)>0$. Let $h(x)$ be a bounded and measurable function. If $\boldsymbol{E}\left(Y^{2} \bar{G}^{-1}(Y)\right)<\infty$ and $\boldsymbol{E} g^{2}\left(\theta_{0}^{\tau} X\right)<\infty$, then

$$
\sqrt{n}\left(Z_{n}(b)-\mu(b)+\mu_{g}\right) \rightarrow N\left(0, \sigma_{b}^{2}\right) \text { in distribution as } n \rightarrow \infty
$$

where

$$
\begin{aligned}
\sigma_{b}^{2}= & \int \frac{\phi_{b}^{2}(x, y)}{\bar{G}(y)} F^{0}(\mathrm{~d} x, \mathrm{~d} y)-\int \frac{\psi_{b}^{2}(s)}{\bar{F}(s) \bar{G}^{2}(s)} \mathrm{d} G(s) \\
& -\mu^{2}(b)-2 \alpha(b)+2 \mu_{g} \mu(b)+m_{g}^{2}-\mu_{g}^{2} .
\end{aligned}
$$


Proof of Lemma D.1 Define $\beta_{n}=\left(n^{-1} \log n\right)^{\frac{3}{4}}, \tilde{\delta}_{i}=I\left(Y_{i}>C_{i}\right)$, and

$$
T_{i}(u)=\frac{\tilde{\delta}_{i} I\left(U_{i} \leq u\right)}{\bar{H}\left(U_{i}\right)}-\int_{-\infty}^{u} \frac{I\left(s \leq U_{i}\right)}{\bar{H}^{2}(s)} \mathrm{d} H^{0}(s) .
$$

By (3.11) of He and Wong [14], we have

$$
\int \phi_{b}(x, y) F_{n}^{0}(\mathrm{~d} x, \mathrm{~d} y)=\frac{1}{n} \sum_{j=1}^{n}\left(\xi_{j}+S_{j}(b)\right)+O_{a . s .}\left(\beta_{n}\right),
$$

where $\xi_{j}(b)=\delta_{j} \phi_{b}\left(X_{j}, U_{j}\right) \bar{G}^{-1}\left(U_{j}\right)$ and $S_{j}(b)=\mathbf{E}\left(\xi_{i}(b) T_{j}\left(U_{i}\right) \mid X_{j}, Y_{j}, C_{j}\right)$, $i \neq j$.

Let $\eta_{i}=g\left(\theta_{0}^{\tau} X_{i}\right) h\left(X_{i}\right)$. Then, $Z_{n}(b)=\frac{1}{n} \sum_{j=1}^{n}\left(\xi_{j}(b)+S_{j}(b)-\eta_{j}\right)+O_{\text {a.s. }}\left(\beta_{n}\right)$. Since $\left\{\xi_{j}(b)+S_{j}(b)-\eta_{j} ; j=1,2, \ldots, n\right\}$ are independent and identically distributed, $\mathbf{E} \xi_{j}(b)=\mu(b), \mathbf{E} S_{j}(b)=0$, and $\mathbf{E} \eta_{j}=\mu_{g}$, it follows from the central limit theorem that $\sqrt{n}\left(Z_{n}(b)-\mu(b)+\mu_{g}\right) \rightarrow N\left(0, \sigma_{b}^{2}\right)$ in distribution, where $\sigma_{b}^{2}=\operatorname{Var}\left(\xi_{i}(b)+S_{i}(b)-\eta_{i}\right)$. From (3.12), (3.13) and (3.14) of He and Wong [14], we have

$$
\operatorname{Var}\left(\xi_{i}(b)+S_{i}(b)\right)=\int \frac{\phi_{b}^{2}(x, y)}{\bar{G}(y)} F^{0}(\mathrm{~d} x, \mathrm{~d} y)-\mu^{2}(b)-\int \frac{\psi_{b}^{2}(s)}{\bar{F}(s) \bar{G}^{2}(s)} \mathrm{d} G(s) .
$$

Also, after some algebraic manipulations, we get $\operatorname{Var}\left(\eta_{i}\right)=m_{g}^{2}-\mu_{g}^{2}$ and $\operatorname{Cov}\left(\xi_{i}(b)+S_{i}(b), \eta_{i}\right)=\alpha(b)-\mu(b) \mu_{g}$. Thus, (D.8) holds.

The following lemmas give the asymptotic properties of $Z_{n}$ of (D.4).

Lemma D.2 Let $F$ and $G$ be continuous, $b_{F} \leq b_{G}$ and $h(x)$ be a bounded and measurable function. If $\boldsymbol{E}^{2}\left(\theta_{0}^{\tau} X\right)<\infty, \boldsymbol{E}\left(\bar{F}^{-1}(C) I\left(C \leq b_{F}\right)\right)<\infty$, and $\boldsymbol{E}\left(Y^{2} \bar{G}^{-1}(Y)\right)<\infty$, then we have

$$
\sqrt{n} Z_{n} \rightarrow N\left(0, \sigma^{2}\right) \quad \text { in distribution, }
$$

where

$$
\sigma^{2}=\int \sigma_{1}^{2}(x) h^{2}(x) f(x) \mathrm{d} x-\int \frac{\psi^{2}(s)}{\bar{F}(s) \bar{G}^{2}(s)} \mathrm{d} G(s),
$$

$\sigma_{1}^{2}(x)=\boldsymbol{E}\left(\left(\varepsilon^{*}\right)^{2} \mid X=x\right), \varepsilon^{*}$ is given in (1.2), and $\psi(s)=\int_{y \geq s} y h(x) F^{0}(\mathrm{~d} x, \mathrm{~d} y)$.

Proof of Lemma D.2 Let $b_{m}<b_{F}$ and $b_{m} \rightarrow b_{F}$ as $m \rightarrow \infty$. Define

$$
\psi_{m}(s)=\int_{y \geq s} \phi_{m}(x, y) F^{0}(\mathrm{~d} x, \mathrm{~d} y) \quad \text { with } \quad \phi_{m}(x, y)=h(x) y I\left(y \leq b_{m}\right) .
$$


Let

$$
\begin{aligned}
\sigma_{m}^{2}= & \int \frac{\phi_{m}^{2}(x, y)}{\bar{G}(y)} F^{0}(\mathrm{~d} x, \mathrm{~d} y)-\int \frac{\psi_{m}^{2}(s)}{\bar{F}(s) \bar{G}^{2}(s)} \mathrm{d} G(s) \\
& -\mu^{2}(m)-2 \alpha(m)+2 \mu_{g} \mu(m)+m_{g}^{2}-\mu_{g}^{2},
\end{aligned}
$$

where $\mu(m), \alpha(m), \mu_{g}$, and $m_{g}^{2}$ are given in (D.5),(D.6), and (D.7).

We first prove that $\sigma_{m}^{2} \rightarrow \sigma^{2}$ as $m \rightarrow \infty$. Under single index model (1.1), by the dominated convergence theorem, we have

$$
\sigma_{m}^{2} \rightarrow \int \frac{y^{2} h^{2}(x)}{\bar{G}(y)} F^{0}(\mathrm{~d} x, \mathrm{~d} y)-\int \frac{\psi^{2}(s)}{\bar{F}(s) \bar{G}^{2}(s)} \mathrm{d} G(s)-m_{g}^{2}
$$

Furthermore, by model (1.2), we have $\sigma_{m}^{2} \rightarrow \mathbf{E}\left(\left(\varepsilon^{*}\right)^{2} h^{2}(X)\right)-\int \frac{\psi^{2}(s)}{\bar{F}(s) \bar{G}^{2}(s)} \mathrm{d} G(s)$. Therefore, we obtain $\sigma_{m}^{2} \rightarrow \sigma^{2}$.

Define

$$
\begin{aligned}
L_{n, m} & =\int \phi_{m}(x, y) \mathrm{d}\left(F_{n}^{0}(x, y)-F^{0}(x, y)\right)-M_{n}+\mu_{g}, \\
K_{n} & =\int y h(x) \mathrm{d}\left(F_{n}^{0}(x, y)-F^{0}(x, y)\right)-M_{n}+\mu_{g} .
\end{aligned}
$$

Note that $Z_{n}=K_{n}$ under single index model (1.1). Then, by Lemma D.1, for each $m, \sqrt{n} L_{n, m}$ converges weakly to $B_{m}$ that has the normal distribution $\sim N\left(0, \sigma_{m}^{2}\right)$. Since $\sigma_{m}^{2} \rightarrow \sigma^{2}$, we see that $B_{m}$ converges in distribution to $N\left(0, \sigma^{2}\right)$ as $m \rightarrow \infty$. By (3.19) of He and Wong [14], we know that $\lim _{m \rightarrow \infty} \limsup _{n \rightarrow \infty} P\left(\sqrt{n}\left|L_{n, m}-Z_{n}\right| \geq \epsilon\right)=0$ for any $\epsilon>0$. Thus, by Theorem 4.2 of Billingsley [1], (D.9) holds.

Recall $\mathcal{H}$ defined in Sect. 5.

Lemma D.3 Let $F$ and $G$ be continuous, $b_{F} \leq b_{G}$, and $h(x)$ be a bounded and measurable function. Under $\mathcal{H}$, if $\boldsymbol{E}\left(Y^{k} \overline{\bar{G}}^{-1}(Y)\right)<\infty, k=1,2$, and $\boldsymbol{E} g^{2}\left(\theta_{0}^{\tau} X\right)<\infty$, then we have

$$
\limsup _{n \rightarrow \infty} \sqrt{\frac{n}{2 \ln \ln n}}\left|Z_{n}\right| \rightarrow \sigma, \quad \text { a.s. }
$$

where $\sigma^{2}$ is given in (D.10).

Proof of Lemma D.3 The proof of the lemma is similar to that of Theorem 2.1 of He and Wang [13].

Acknowledgements This research was supported by the National Natural Science Foundation of China Grant 10231030 and RFDP, and grants from the Research Grants Council of the Hong Kong Special Administrative Region, China (Project No. HKU 7323/01M and HKBU7058/05P). 
The authors would like to thank the editor and referees for their constructive comments and suggestions which led to a significant improvement of the presentation. The first draft of the paper was finished when the corresponding author was at the University of Hong Kong.

\section{References}

1. Billingsley, P.: Convergence of Probability Measures. Wiley, New York (1968)

2. Carroll, R.J., Fan, J. Q., Gijbels, I., and Wand, M. P.: Generalized partially linear single-index model. J. Am. Stat. Assoc. 92, 477-489 (1997)

3. Chen, K., Lo, S.H.: On the rate of uniform convergence of the product-limit estimator : Strong and weak laws. Ann. Stat. 25, 1050-1087 (1997)

4. Daniela, C., Jeffrey, H., Lépold, S.: Automatic smoothing and estimation in single index poisson regression. J. Nonpanametr. Stat. 14, 307-323 (2002)

5. Delecroix, M., Härdle, W., and Hristache, M.: Efficient estimation in conditional single index regression. J. Multi. Anal. 86, 213-226 (2003)

6. Fan, J., Gijbels, I.: Censored regression: local linear approximations and their applications. J. Am. Stat. Assoc. 89, 560-570 (1994)

7. Friedman, J.H., Stuetzle, W.: Projection pursuit regression. J. Am. Stati. Assoc. 76, 817-823 (1981)

8. Gill, R.D.: Large sample behavior of the product limit estimator on the whole line. Ann. Stat. 11, 49-58 (1983)

9. Gu, M., Lai, L.: Functional laws of the iterated logarithm for the product limit estimator of a distribution function under random censorship or truncation. Ann. Probab. 18, 160-189 (1990)

10. Hall, P.: On projection pursuit regression. Ann. Statist. 17, 573-588 (1989)

11. Härdle, W., Hall, P., Ichimura, H.: Optimal smoothing in single-index models. Ann. Stat. 21, 157-178 (1993)

12. Härdle, W., Stoker, T.: Investigating smooth multiple regression by the method of averrage derivatives. J. Am. Stat. Assoc. 84, 986-995 (1989)

13. He, S.Y., Yang, G.: Estimation of the truncation probability in the random truncation model. Ann. Stat. 26, 1011-1027 (1998)

14. He, S.Y., Wong, H.: The central limit theorem of linear regression model under right censorship. Sci. China Ser. A. 2003, (to appear) (2003)

15. He, S.Y., Wang, Y.H.: The law of the iterated logarithm of the Kaplan-Meier integral and its application. Chin. Ann. Math. 25B, 199-206 (2004)

16. Horowitz, J., Härdle, W.: Direct Semiparametric estimation of single-index models with discrete covariates. J. Am. Stat. Assoc. 91, 1632-1640 (1996)

17. Huber, P.: Projection pursuit (with discussion). Ann. Statist. 13, 435-475 (1985)

18. Huh, J., Park, B.U.: Likelihood-based local polynomial fitting for single-index models. J. Multi. Anal. 80, 302-321 (2002)

19. Ichimura, H.: Estimation of single-index models. PhD dissertation, Department of. Economics, MIT (1987)

20. Ichimura, H.: Semiparametric least squares (SLS) and weighted SLS estimation of single-index models. J. Econom 58, 71-120 (1993)

21. Koul, H., Susarla, V., Van Ryzin, J.: Regression analysis with randomly right censored data. Ann. Stat. 9, 1276-1288 (1981)

22. Leurgans, S.: Linear models, random censoring, and synthetic data. Biometrika 74, 301-309 (1987)

23. Li, K.C., Duan, N.: Regression analysis under link violation. Ann. Stat. 17, 1009-1052 (1989)

24. Li, K.C., Wang, J.L., Chen, C.: Dimension reduction for censored regression data. Ann. Stat. 27, 1-23 (1999)

25. Stute, W., Wang, J.L.: The strong law under random censorship. Ann. Stat. 21, 1591-1607 (1993)

26. Stute, W.: The central limit theorem under random censorship. Ann. Stat. 23, $422-439$ (1995)

27. Stute, W. (1999). Nonlinear censored regression. Stat. Sinica 9. 1089-1102 (1999)

28. Wang, Y.H., He, S.Y., Zhu, L.X., Yuen, K.C.:Asymptotics for a single-index model under random censorship. Research Report Serial No.354, Department of Statistics and Actuarial Science, the University of Hong Kong (2005) 
29. Xia, Y., Tong, H., Li, W.K.:On extended partially linear single-index models. Biometrika 86, 831-842 (1999)

30. Zhou, M.: Some properties of the Kaplan Meier estimator for indenpendent nonidentically distributed random variables. Ann. Stat. 19, 2266-2274 (1991)

31. Zhu, L.X., Fang, K.T.: On projection pursuit approximation for nonparametric regression.In: Proceedings. of Order Statistic. and Nonparametrics: Theory and Applications, pp. 455-469 (1992) 